\title{
On the two-dimensional hyperbolic stochastic sine-Gordon equation
}

\author{
Tadahiro $\mathrm{Oh}^{1,2}$ (D) Tristan Robert ${ }^{1,2,3} \cdot$ Philippe Sosoe $^{4} \cdot$ Yuzhao Wang $^{5}$
}

Received: 11 August 2019 / Revised: 29 December 2019 / Published online: 5 February 2020

(c) The Author(s) 2020

\begin{abstract}
We study the two-dimensional stochastic sine-Gordon equation (SSG) in the hyperbolic setting. In particular, by introducing a suitable time-dependent renormalization for the relevant imaginary Gaussian multiplicative chaos, we prove local wellposedness of SSG for any value of a parameter $\beta^{2}>0$ in the nonlinearity. This exhibits sharp contrast with the parabolic case studied by Hairer and Shen (Commun Math Phys 341(3):933-989, 2016) and Chandra et al. (The dynamical sine-Gordon model in the full subcritical regime, arXiv:1808.02594 [math.PR], 2018), where the parameter is restricted to the subcritical range: $0<\beta^{2}<8 \pi$. We also present a triviality result for the unrenormalized SSG.
\end{abstract}

Keywords Stochastic sine-Gordon equation - Sine-Gordon equation ·

Renormalization · White noise · Gaussian multiplicative chaos

Tadahiro Oh

hiro.oh@ed.ac.uk

Tristan Robert

trobert@math.uni-bielefeld.de

Philippe Sosoe

psosoe@math.cornell.edu

Yuzhao Wang

y.wang.14@bham.ac.uk

1 School of Mathematics, The University of Edinburgh, James Clerk Maxwell Building, The King's Buildings, Peter Guthrie Tait Road, Edinburgh EH9 3FD, UK

2 The Maxwell Institute for the Mathematical Sciences, James Clerk Maxwell Building, The King's Buildings, Peter Guthrie Tait Road, Edinburgh EH9 3FD, UK

3 Present Address: Fakultät für Mathematik, Universität Bielefeld, Postfach 1001 31, 33501 Bielefeld, Germany

4 Department of Mathematics, Cornell University, 584 Malott Hall, Ithaca, NY 14853, USA

5 School of Mathematics, University of Birmingham, Watson Building, Edgbaston, Birmingham B15 2TT, UK 


\section{Mathematics Subject Classification 35L71 · 60H15}

\section{Contents}

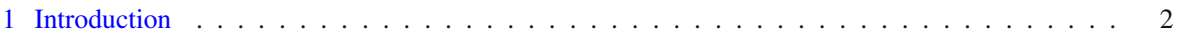

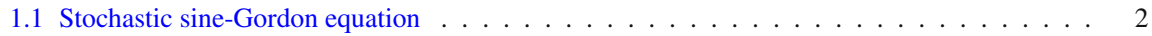

1.2 Renormalization of the nonlinearity . . . . . . . . . . . . . . . . 5

1.3 Main results . . . . . . . . . . . . . . . . . . . . . . . 7

2 On the imaginary Gaussian multiplicative chaos . . . . . . . . . . . . . . . . . 12

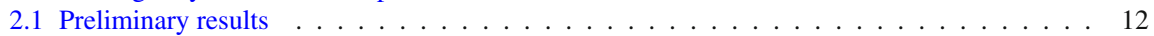

2.2 Estimates on the stochastic objects . . . . . . . . . . . . . . . . . . 18

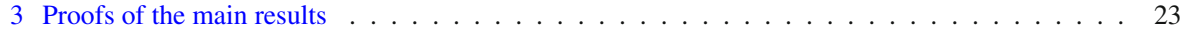

3.1 Strichartz estimates and other useful lemmas . . . . . . . . . . . . . . . . . . . 23

3.2 Local well-posedness . . . . . . . . . . . . . . . . . . . . . . . . . . . . . 25

3.3 Triviality of the unrenormalized model . . . . . . . . . . . . . . . . . . . . . . . . 29

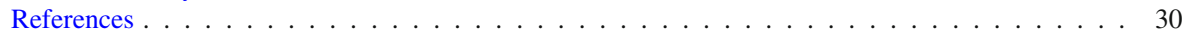

\section{Introduction}

\subsection{Stochastic sine-Gordon equation}

We consider the following hyperbolic stochastic sine-Gordon equation on $\mathbb{T}^{2}=$ $(\mathbb{R} / 2 \pi \mathbb{Z})^{2}$ with an additive space-time white noise forcing:

$$
\left\{\begin{array}{l}
\partial_{t}^{2} u+(1-\Delta) u+\gamma \sin (\beta u)=\xi \\
\left.\left(u, \partial_{t} u\right)\right|_{t=0}=\left(u_{0}, u_{1}\right)
\end{array} \quad(t, x) \in \mathbb{R}_{+} \times \mathbb{T}^{2},\right.
$$

where $\gamma$ and $\beta$ are non-zero real numbers and $\xi(t, x)$ denotes a (Gaussian) space-time white noise on $\mathbb{R}_{+} \times \mathbb{T}^{2}$. In this paper, we are interested in studying the model (1.1), where the linear dynamics is given by the wave equation. ${ }^{1}$

The stochastic nonlinear wave equations (SNLW) have been studied extensively in various settings; see [11, Chapter 13] for the references therein. In recent years, there has been a rapid progress on the theoretical understanding of SNLW with singular stochastic forcing. In [19], Gubinelli, Koch, and the first author studied the following stochastic nonlinear wave equations with an additive space-time white noise on $\mathbb{T}^{2}$ :

$$
\partial_{t}^{2} u+(1-\Delta) u+u^{k}=\xi
$$

where $k \geq 2$ is an integer. The main difficulty of this problem comes from the roughness of the space-time white noise $\xi$, which can already be seen at the linear level. Let $\Psi$ denote the stochastic convolution, solving the linear stochastic wave equation:

\footnotetext{
${ }^{1}$ More precisely, by the Klein-Gordon equation. In the following, we study (1.1) with the Klein-Gordon operator $\partial_{t}^{2}+(1-\Delta)$ to avoid a separate treatment at the zeroth frequency. Note, however, that the same results with inessential modifications also hold for (1.1) with the wave operator $\partial_{t}^{2}-\Delta$. The same comment applies to (1.2) (and (1.3), respectively), which we simply refer to as the stochastic nonlinear (and linear, respectively) wave equation in the following.
} 


$$
\partial_{t}^{2} \Psi+(1-\Delta) \Psi=\xi
$$

For the spatial dimension $d \geq 2$, the stochastic convolution $\Psi$ is not a classical function but is merely a Schwartz distribution. See Sect. 1.2 and Lemma 2.6 below. This causes an issue in making sense of powers $\Psi^{k}$ and a fortiori of the full nonlinearity $u^{k}$ in (1.2), necessitating a renormalization of the nonlinearity. In [19], by introducing an appropriate time-dependent renormalization, the authors proved local well-posedness of (a renormalized version of) (1.2) on $\mathbb{T}^{2}$. See [20,21,33,35,37,39] for related works on nonlinear wave equations with singular stochastic forcing and/or rough random initial data. We point out that these works handle polynomial nonlinearities.

Our main goal in this paper is to extend the analysis to SNLW with a non-polynomial nonlinearity, of which trigonometric functions are the simplest. As in the case of a polynomial nonlinearity, a proper renormalization needs to be introduced to our problem. This can be seen from the regularity of the stochastic convolution $\Psi$ as above. More informally, we can see the necessity of a renormalization from the fast oscillation of $\sin (\beta u)$ due to the (expected) roughness of $u$, which makes the nonlinearity $\sin (\beta u)$ tend to 0 in some limiting sense. See Proposition 1.4. In order to counterbalance such decay and have a non-trivial solution, we will take $\gamma \rightarrow \infty$ in (1.1). See Sect. 1.2.

The main new difficulty comes from the non-polynomial nature of the nonlinearity, which makes the analysis of the relevant stochastic object particularly non-trivial (as compared to the previous work [19]). In particular, after introducing a time-dependent renormalization, we show that the regularity of the main stochastic terms depends on both the parameter $\beta \in \mathbb{R} \backslash\{0\}$ and the time $t>0$. See Proposition 1.1. This is a striking difference from the polynomial case.

The sine nonlinearity in (1.1) is closely related to models arising from both relativistic and quantum field theories $[4,13,40]$ and has attracted a lot of attention over the past years. Moreover, the following (deterministic) one-dimensional sine-Gordon equation:

$$
\partial_{t}^{2} u-\partial_{x}^{2} u+\sin u=0
$$

is known to be completely integrable, to which a vast literature was devoted. Here, we simply mention the work by McKean [31,32]. See the references therein. In particular, in [32], McKean constructed an invariant Gibbs measure for (1.4).

In the one-dimensional case, the stochastic convolution $\Psi$ in (1.3) has (spatial) regularity $\frac{1}{2}-\varepsilon, \varepsilon>0$, and thus a straightforward computation yields local wellposedness of (1.1). The situation becomes much more delicate in the two-dimensional setting. In the parabolic setting, Hairer and Shen [24] and Chandra et al. [8] studied the following parabolic sine-Gordon model on $\mathbb{T}^{2}$ :

$$
\partial_{t} u-\frac{1}{2} \Delta u+\gamma \sin (\beta u)=\xi
$$

In particular, they observed that the difficulty of the problem depends sensitively on the value of $\beta^{2}>0$. At a heuristic level, this model is comparable to various models appearing in constructive quantum field theory, where the dimension $d$ can 
be expressed in terms of the parameter $\beta$; for example, the $\Phi_{d}^{3}$-model (and the $\Phi_{d^{-}}^{4}$ model, respectively) formally corresponds to (1.5) with $d=2+\frac{\beta^{2}}{2 \pi}$ (and $d=2+\frac{\beta^{2}}{4 \pi}$, respectively). In terms of the actual well-posedness theory, the Da Prato-Debussche trick [10] along with a standard Wick renormalization yields local well-posedness of (1.5) for $0<\beta^{2}<4 \pi$. It turns out that there is an infinite number of thresholds: $\beta^{2}=\frac{j}{j+1} 8 \pi, j \in \mathbb{N}$, where one encounters new divergent stochastic objects, requiring further renormalizations. By using the theory of regularity structures [22], Hairer and Shen [24] proved local well-posedness up to the second threshold $\beta^{2}<\frac{16 \pi}{3}$ in the first work. In the second work [8], together with Chandra, they pushed the local well-posedness theory to the entire subcritical regime $\beta^{2}<8 \pi$. When $\beta^{2}=8 \pi$, the Eq. (1.5) is critical and falls outside the scope of the current theory. In fact, it is expected that, for $\beta^{2} \geq 8 \pi$, any reasonable approach would yield a trivial solution, solving the linear stochastic heat equation. The Eq. (1.5) possesses a formally invariant Gibbs measure, and thus it is expected that Bourgain's invariant measure argument [6,7] would allow one to extend the local-in-time dynamics constructed in $[8,24]$ globally in time. We mention a recent work [29] on the construction of a Gibbs measure for the sine-Gordon model with a log-correlated Gaussian process (but their result is restricted to $d=1$ ). We also mention recent papers $[14,43]$ on dynamical problems with an exponential nonlinearity in the two-dimensional setting; in [14], Garban studied the dynamical problem with an exponential nonlinearity in the parabolic setting, while in [43], Sun and Tzvetkov considered a dispersion generalized nonlinear wave equation with an exponential nonlinearity in the context of random data well-posedness theory. ${ }^{2}$

Our model (1.1) in the hyperbolic setting is sensitive to the value of $\beta^{2}>0$ as in the parabolic case. Furthermore, due to the non-conservative nature of the problem, ${ }^{3}$ the renormalization we employ is time-dependent as in [19] and the difficulty also depends on the time parameter $t>0$. See Proposition 1.1. On the one hand, by taking $t>0$ small, we can make sure that the relevant stochastic object is not too rough, allowing us to establish local well-posedness of (1.1) for any value of $\beta^{2}>0$ (Theorem 1.2). On the other hand, even if we start the dynamics with small $\beta^{2}>0$, our analysis, when compared to the parabolic setting $[8,24]$, formally indicates existence of an infinite number of thresholds $T_{j}=T_{j}(\beta)$, now given in terms of time,

$$
T_{j}=\frac{16 j \pi}{(j+1) \beta^{2}}, \quad j \in \mathbb{N},
$$

where we encounter new divergent stochastic objects, requiring further renormalizations. As in the parabolic case, the time $T_{\infty}=\frac{16 \pi}{\beta^{2}}$ corresponds to the critical value, after which we do not expect to be able to extend the dynamics. ${ }^{4}$ It is quite intriguing that the singular nature of the problem (1.1) depends sensitively on time and gets worse over time, contrary to the parabolic setting.

\footnotetext{
2 See also a recent preprint [38], where we studied SNLW on $\mathbb{T}^{2}$ with the exponential nonlinearity.

3 We are considering the problem without the damping term, where there is no invariant measure for the linear dynamics. See Remark 1.5 below.

4 Or perhaps, the dynamics may trivialize to the linear dynamics after the critical time $T_{\infty}$.
} 


\subsection{Renormalization of the nonlinearity}

In order to explain the renormalization procedure, we first consider the following regularized equation for (1.1):

$$
\partial_{t}^{2} u_{N}+(1-\Delta) u_{N}+\gamma \operatorname{Im}\left(e^{i \beta u_{N}}\right)=\mathbf{P}_{N} \xi
$$

where $\mathbf{P}_{N}$ is a smooth frequency projector onto the (spatial) frequencies $\left\{n \in \mathbb{Z}^{2}\right.$ : $|n| \leq N\}$, associated with a Fourier multiplier

$$
\chi_{N}(n)=\chi\left(N^{-1} n\right)
$$

for some fixed non-negative function $\chi \in C_{c}^{\infty}\left(\mathbb{R}^{2}\right)$ with supp $\chi \subset\left\{\xi \in \mathbb{R}^{2}:|\xi| \leq 1\right\}$ and $\chi \equiv 1$ on $\left\{\xi \in \mathbb{R}^{2}:|\xi| \leq \frac{1}{2}\right\}$.

We first define the truncated stochastic convolution $\Psi_{N}=\mathbf{P}_{N} \Psi$, solving the truncated linear stochastic wave equation:

$$
\partial_{t}^{2} \Psi_{N}+(1-\Delta) \Psi_{N}=\mathbf{P}_{N} \xi
$$

with the zero initial data. With $\langle\cdot\rangle=\left(1+|\cdot|^{2}\right)^{\frac{1}{2}}$, let $S(t)$ denote the linear wave propagator

$$
S(t)=\frac{\sin (t\langle\nabla\rangle)}{\langle\nabla\rangle},
$$

defined as a Fourier multiplier operator. Namely, we set

$$
S(t) f=\sum_{n \in \mathbb{Z}^{2}} \frac{\sin (t\langle n\rangle)}{\langle n\rangle} \widehat{f}(n) e_{n},
$$

where $\widehat{f}(n)$ is the Fourier coefficient of $f$ and $e_{n}(x)=(2 \pi)^{-1} e^{i n \cdot x}$ as in (1.26). Then, the truncated stochastic convolution $\Psi_{N}$, solving (1.8), is given by

$$
\Psi_{N}(t)=\int_{0}^{t} S\left(t-t^{\prime}\right) \mathbf{P}_{N} d W\left(t^{\prime}\right)
$$

where $W$ denotes a cylindrical Wiener process on $L^{2}\left(\mathbb{T}^{2}\right)$ :

$$
W(t) \stackrel{\text { def }}{=} \sum_{n \in \mathbb{Z}^{2}} B_{n}(t) e_{n}
$$

and $\left\{B_{n}\right\}_{n \in \mathbb{Z}^{2}}$ is defined by $B_{n}(0)=0$ and $B_{n}(t)=\left\langle\xi, \mathbf{1}_{[0, t]} \cdot e_{n}\right\rangle_{t, x}$. Here, $\langle\cdot, \cdot\rangle_{t, x}$ denotes the duality pairing on $\mathbb{R} \times \mathbb{T}^{2}$ and thus we formally have

$$
B_{n}(t)=\left\langle\xi, \mathbf{1}_{[0, t]} e_{n}\right\rangle_{t, x}=“ \int_{0}^{t} \int_{\mathbb{T}^{2}} \overline{e_{n}(x)} \xi\left(d x d t^{\prime}\right) "
$$


As a result, we see that $\left\{B_{n}\right\}_{n \in \mathbb{Z}^{2}}$ is a family of mutually independent complex-valued ${ }^{5}$ Brownian motions conditioned so that $B_{-n}=\overline{B_{n}}, n \in \mathbb{Z}^{2}$. By convention, we normalized $B_{n}$ such that $\operatorname{Var}\left(B_{n}(t)\right)=t$. Then, for each fixed $x \in \mathbb{T}^{2}$ and $t \geq 0$, we see that $\Psi_{N}(t, x)$ is a mean-zero real-valued Gaussian random variable with variance

$$
\begin{aligned}
\sigma_{N}(t) & \stackrel{\text { def }}{=} \mathbb{E}\left[\Psi_{N}(t, x)^{2}\right]=\frac{1}{4 \pi^{2}} \sum_{n \in \mathbb{Z}^{2}} \chi_{N}^{2}(n) \int_{0}^{t}\left[\frac{\sin \left(\left(t-t^{\prime}\right)\langle n\rangle\right)}{\langle n\rangle}\right]^{2} d t^{\prime} \\
& =\frac{1}{4 \pi^{2}} \sum_{n \in \mathbb{Z}^{2}} \chi_{N}^{2}(n)\left\{\frac{t}{2\langle n\rangle^{2}}-\frac{\sin (2 t\langle n\rangle)}{4\langle n\rangle^{3}}\right\} \sim t \log N
\end{aligned}
$$

for all $t \in[0,1]$ and $N \gg 1$. We emphasize that the variance $\sigma_{N}(t)$ is time-dependent. For any $t>0$, we see that $\sigma_{N}(t) \rightarrow \infty$ as $N \rightarrow \infty$, showing that $\left\{\Psi_{N}(t)\right\}_{N \in \mathbb{N}}$ is almost surely unbounded in $W^{0, p}\left(\mathbb{T}^{2}\right)$ for any $1 \leq p \leq \infty$. See also Lemma 2.6 below.

If we were to proceed with a Picard iteration to study (1.6), the next term we need to study is

$$
e^{i \beta \Psi_{N}}=\sum_{k=0}^{\infty} \frac{(i \beta)^{k}}{k !} \Psi_{N}^{k} .
$$

As pointed above, the power $\Psi_{N}^{k}, k \geq 2$, does not have any nice limiting behavior as $N \rightarrow \infty$. As in [19], we now introduce the Wick renormalization:

$$
: \Psi_{N}^{k}(t, x): \stackrel{\text { def }}{=} H_{k}\left(\Psi_{N}(t, x) ; \sigma_{N}(t)\right)
$$

to each power $\Psi_{N}^{k}$ appearing in (1.13). Here, $H_{k}$ denotes the $k$ th Hermite polynomial, defined through the generating function:

$$
e^{t x-\frac{\sigma}{2} t^{2}}=\sum_{k=0}^{\infty} \frac{t^{k}}{k !} H_{k}(x ; \sigma) .
$$

From (1.14) and (1.15), the renormalized complex exponential is then given by

$$
\begin{aligned}
\Theta_{N}(t, x) & =: e^{i \beta \Psi_{N}(t, x)}: \stackrel{\text { def }}{=} \sum_{k=0}^{\infty} \frac{(i \beta)^{k}}{k !}: \Psi_{N}^{k}(t, x): \\
& =e^{\frac{\beta^{2}}{2} \sigma_{N}(t)} e^{i \beta \Psi_{N}(t, x)} .
\end{aligned}
$$

Following [8], we refer to $\Theta_{N}$ as the imaginary Gaussian multiplicative chaos. The following proposition establishes the regularity and convergence property of the imaginary Gaussian multiplicative chaos $\Theta_{N}$.

\footnotetext{
5 In particular, $B_{0}$ is a standard real-valued Brownian motion.
} 
Proposition 1.1 Let $\beta \in \mathbb{R} \backslash\{0\}$ and $T>0$ such that $\beta^{2} T<8 \pi$. Then, given any finite $p, q \geq 1$ and any $\alpha>0$ satisfying $\alpha>\frac{\beta^{2} T}{8 \pi}$, the sequence of random variables $\Theta_{N}$ is a Cauchy sequence in $L^{p}\left(\Omega ; L^{q}\left([0, T] ; W^{-\alpha, \infty}\left(\mathbb{T}^{2}\right)\right)\right)$ and hence converges to some limit $\Theta$ in $L^{p}\left(\Omega ; L^{q}\left([0, T] ; W^{-\alpha, \infty}\left(\mathbb{T}^{2}\right)\right)\right)$.

In view of the convergence of the truncated stochastic convolution $\Psi_{N}$ to $\Psi$ (see Lemma 2.6), it is natural to write the limit $\Theta$ as

$$
\Theta=: e^{i \beta \Psi}: .
$$

See Remark 1.3 below on the uniqueness of the limiting process $\Theta$.

In the stationary and parabolic settings, analogous results were established by Lacoin et al. [28, Theorem 3.1] and Hairer and Shen [24, Theorem 2.1]. ${ }^{6}$ The main difference between Proposition 1.1 and the previous results in [24,28] is the dependence of the regularity on the time parameter $T>0$. In particular, as $T$ increases, the regularity of $\Theta_{N}$ gets worse. On the other hand, for fixed $\beta \in \mathbb{R} \backslash\{0\}$, by taking $T>0$ small, we can take $\left\{\Theta_{N}(t)\right\}_{N \in \mathbb{N}}$ almost bounded in $L^{2}\left(\mathbb{T}^{2}\right), 0<t \leq T$.

The proof of Proposition 1.1 is in the spirit of [24,28]. In the case of a polynomial nonlinearity $[19,20]$, it is enough to estimate the second moment and then invoke the Wiener chaos estimate (see, for example, Lemma 2.5 in [20]) to obtain the $p$ th moment bound, since the stochastic objects in $[19,20]$ all belong to Wiener chaoses of finite order. The imaginary Gaussian multiplicative chaos $\Theta_{N}$ in (1.16), however, does not belong to any Wiener chaos of finite order. This forces us to estimate all its higher moments by hand. In Sect. 2, we present a proof of Proposition 1.1. While we closely follow the argument in [24], our argument is based on an elementary calculation. See Lemma 2.5.

\subsection{Main results}

In view of the previous discussion, we are thus led to study the following renormalized stochastic sine-Gordon equation:

$$
\left\{\begin{array}{l}
\partial_{t}^{2} u_{N}+(1-\Delta) u_{N}+\gamma_{N} \sin \left(\beta u_{N}\right)=\mathbf{P}_{N} \xi \\
\left.\left(u_{N}, \partial_{t} u_{N}\right)\right|_{t=0}=\left(u_{0}, u_{1}\right),
\end{array}\right.
$$

where $\gamma_{N}$ is defined by

$$
\gamma_{N}(t, \beta)=e^{\frac{\beta^{2}}{2} \sigma_{N}(t)} \longrightarrow \infty,
$$

as $N \rightarrow \infty$. We now state the local well-posedness result.

Theorem 1.2 Let $\beta \in \mathbb{R} \backslash\{0\}$ and $s>0$. Given any $\left(u_{0}, u_{1}\right) \in \mathcal{H}^{s}\left(\mathbb{T}^{2}\right)=H^{s}\left(\mathbb{T}^{2}\right) \times$ $H^{s-1}\left(\mathbb{T}^{2}\right)$, the Cauchy problem (1.17) is uniformly locally well-posed in the following

\footnotetext{
${ }^{6}$ While Theorem 2.1 in [24] is stated in terms of space-time regularity, it implies that the conclusion of Proposition 1.1 holds in the parabolic case, provided that $\beta^{2}<4 \pi$ and $\alpha>\frac{\beta^{2}}{4 \pi}$, i.e. corresponding to the restrictions on $\beta$ and $\alpha$ in Proposition 1.1 with $T=2$.
} 
sense; there exists $T_{0}=T_{0}\left(\left\|\left(u_{0}, u_{1}\right)\right\|_{\mathcal{H}^{s}}, \beta\right)>0$ such that given any $0<T \leq T_{0}$ and $N \in \mathbb{N}$, there exists a set $\Omega_{N}(T) \subset \Omega$ such that

(i) for any $\omega \in \Omega_{N}(T)$, there exists a unique solution $u_{N}$ to (1.17) in the class

$$
\Psi_{N}+X^{\sigma}(T) \subset C\left([0, T] ; H^{-\varepsilon}\left(\mathbb{T}^{2}\right)\right),
$$

for any small $\varepsilon>0$, where $\Psi_{N}$ is as in (1.10), $X^{\sigma}(T)$ is the Strichartz space defined in (3.2), and $\sigma=\min (s, 1-\varepsilon)$,

(ii) there exists a uniform estimate on the probability of the complement of $\Omega_{N}(T)$ :

$$
P\left(\Omega_{N}(T)^{c}\right) \longrightarrow 0
$$

uniformly in $N \in \mathbb{N}$, as $T \rightarrow 0$,

Furthermore, there exist an almost surely positive stopping time $\tau=\tau\left(\left\|\left(u_{0}, u_{1}\right)\right\|_{\mathcal{H}^{s}}\right.$, $\beta$ ) and a stochastic process $u$ in the class

$$
\Psi+X^{\sigma}(T) \subset C\left([0, T] ; H^{-\varepsilon}\left(\mathbb{T}^{2}\right)\right)
$$

for any $\varepsilon>0$ such that, given any small $T>0$, on the event $\{\tau \geq T\}$, the solution $u_{N}$ to $(1.17)$ converges in probability to $u$ in $C\left([0, T] ; H^{-\varepsilon}\left(\mathbb{T}^{2}\right)\right)$.

Note that, in Theorem 1.2 above, we can take the parameter $\beta^{2}>0$ arbitrarily large. This exhibits sharp contrast with the parabolic case studied in [8,24], where the parameter was restricted to the subcritical range: $0<\beta^{2}<8 \pi$.

The main point is that Proposition 1.1 shows that the imaginary Gaussian multiplicative chaos $\Theta_{N}$ is almost a function (namely, $\alpha>0$ small in Proposition 1.1) by taking $T=T(\beta)>0$ sufficiently small. In particular, we can apply the analysis from [19] on the polynomial nonlinearity via the Da Prato-Debussche trick to study (1.17); namely write a solution $u_{N}$ to (1.17) as

$$
u_{N}=\Psi_{N}+v_{N}
$$

In view of (1.16) and (1.18), the residual term $v_{N}$ then satisfies

$$
\left\{\begin{array}{l}
\partial_{t}^{2} v_{N}+(1-\Delta) v_{N}+\operatorname{Im}\left(\Theta_{N} e^{i \beta v_{N}}\right)=0 \\
\left.\left(v_{N}, \partial_{t} v_{N}\right)\right|_{t=0}=\left(u_{0}, u_{1}\right)
\end{array}\right.
$$

Then, by taking $T=T(\beta)>0$ sufficiently small, thus guaranteeing the regularity of $\Theta_{N}$ via Proposition 1.1, the standard Strichartz analysis as in [19] along with the fractional chain rule (Lemma 3.2) suffices to conclude Theorem 1.2. As in [19], our argument shows that $u$ constructed in Theorem 1.2 has a particular structure $u=\Psi+v$, where $v \in X^{\sigma}(T)$ satisfies

$$
\left\{\begin{array}{l}
\partial_{t}^{2} v+(1-\Delta) v+\operatorname{Im}\left(\Theta e^{i \beta v}\right)=0 \\
\left.\left(v, \partial_{t} v\right)\right|_{t=0}=\left(u_{0}, u_{1}\right)
\end{array}\right.
$$


where $\Theta$ is the limit of $\Theta_{N}$ constructed in Proposition 1.1.

Next, we consider the stochastic sine-Gordon equation (1.1) without renormalization by studying its frequency-truncated version ${ }^{7}$ :

$$
\left\{\begin{array}{l}
\partial_{t}^{2} u_{N}+(1-\Delta) u_{N}+\sin \left(\beta u_{N}\right)=\mathbf{P}_{N} \xi \\
\left.\left(u_{N}, \partial_{t} u_{N}\right)\right|_{t=0}=\left(u_{0}, u_{1}\right)
\end{array}\right.
$$

In studying the limit as $N \rightarrow \infty$, we expect the solution $u_{N}$ to become singular. As a result, in view of faster and faster "oscillation", we expect $\sin \left(\beta u_{N}\right)$ to tend to 0 as a space-time distribution. This is the reason why we needed to insert a diverging multiplicative constant $\gamma_{N}$ in the renormalized model (1.17).

Remark 1.3 In Proposition 1.1 and Theorem 1.2 above, we used a smooth frequency projector $\mathbf{P}_{N}$ with the multiplier $\chi_{N}$ in (1.7). As in the parabolic case, it is possible to show that the limiting process $\Theta$ of $\Theta_{N}$ in Proposition 1.1 and the limit $u$ of $u_{N}$ in Theorem 1.2 are independent of the choice of the smooth cutoff function $\chi$. See [34] for such an argument in the wave case (with a polynomial nonlinearity). Moreover, we may also proceed by smoothing via a mollification and obtain analogous results. In this case, the limiting processes agree with those constructed in Proposition 1.1 and Theorem 1.2.

In the following, we study the limiting behavior of $u_{N}$, solving the unrenormalized model (1.21) with regularized noises, and establish a triviality result. The heuristics above indicates that the nonlinearity $\sin \left(\beta u_{N}\right)$ tends to 0 in some suitable sense, indicating that $u_{N}$ converges to a solution to the linear stochastic wave equation. The next proposition shows that this is indeed the case.

Proposition 1.4 Let $\beta \in \mathbb{R} \backslash\{0\}$ and fix $\left(u_{0}, u_{1}\right) \in \mathcal{H}^{s}\left(\mathbb{T}^{2}\right)$ for some $s>0$. Given any small $T>0$, the solution $u_{N}$ to (1.21) converges in probability to the solution $u$, satisfying the following linear stochastic wave equation:

$$
\left\{\begin{array}{l}
\partial_{t}^{2} u+(1-\Delta) u=\xi \\
\left.\left(u, \partial_{t} u\right)\right|_{t=0}=\left(u_{0}, u_{1}\right)
\end{array}\right.
$$

in the class $C\left([0, T] ; H^{-\varepsilon}\left(\mathbb{T}^{2}\right)\right), \varepsilon>0$, as $N \rightarrow \infty$.

We point out that the nature of "triviality" in Proposition 1.4 is slightly different from that in the work $[23,33,35]$ with the cubic nonlinearity, where solutions $u_{N}$ to the unrenormalized equation with regularized noises tend to 0 as we remove the regularization. This difference comes from the different renormalization procedures; our renormalization for the sine-Gordon model appears as a multiplication by the renormalization constant. On the other hand, in the case of the cubic nonlinearity, the renormalization is implemented by insertion of a linear term $\gamma_{N} u_{N}$ with a suitable divergent renormalization constant $\gamma_{N}$, thus modifying the linear part of the equation.

\footnotetext{
7 Here, we set $\gamma=1$ in (1.1) and (1.6) for simplicity.
} 
In particular, in the case of the wave equation the modified linear propagator introduces faster and faster oscillations and thus the contribution from the deterministic initial data $\left(u_{0}, u_{1}\right)$ also vanishes. See $[33,35]$.

Following the previous work $[23,33,35]$, the main idea for proving Proposition 1.4 is to artificially insert the renormalization constant $\gamma_{N}$ in the unrenormalized equation (1.21). By using the decomposition $u_{N}=\Psi_{N}+v_{N}$ as before, we have

$$
\left\{\begin{array}{l}
\partial_{t}^{2} v_{N}+(1-\Delta) v_{N}+\gamma_{N}^{-1} \operatorname{Im}\left(\Theta_{N} e^{i \beta v_{N}}\right)=0 \\
\left.\left(v_{N}, \partial_{t} v_{N}\right)\right|_{t=0}=\left(u_{0}, u_{1}\right)
\end{array}\right.
$$

Proposition 1.4 then follows from essentially repeating the proof of Theorem 1.2 along with the asymptotic behavior (1.18) of the renormalization constant.

Remark 1.5 In the case of a polynomial nonlinearity, the local-in-time analysis in [19] essentially applies to the following hyperbolic $\Phi_{2}^{2 k+2}$-model ${ }^{8}$ :

$$
\partial_{t}^{2} u+\partial_{t} u+(1-\Delta) u+u^{2 k+1}=\sqrt{2} \xi
$$

with random initial data distributed by the Gibbs measure $\left(=\Phi_{2}^{2 k+2}\right.$-measure). Then, Bourgain's invariant measure argument [6,7] allows us to establish almost sure global well-posedness of (a renormalized version of) (1.23) and invariance of the Gibbs measure. See [21,37].

By adding the damping term $\partial_{t} u$ to (1.1), we obtain the following "canonical" stochastic sine-Gordon model:

$$
\partial_{t}^{2} u+\partial_{t} u+(1-\Delta) u+\gamma \sin (\beta u)=\sqrt{2} \xi
$$

This equation is a hyperbolic counterpart of the stochastic quantization equation for the quantum sine-Gordon model; the Eq. (1.24) formally preserves the Gibbs measure $\mu$ given by ${ }^{9}$

$d \mu=Z^{-1} \exp \left(-\frac{1}{2} \int_{\mathbb{T}^{2}}|\langle\nabla\rangle u|^{2} d x-\frac{1}{2} \int_{\mathbb{T}^{2}}\left(\partial_{t} u\right)^{2} d x+\frac{\gamma}{\beta} \int_{\mathbb{T}^{2}} \cos (\beta u) d x\right) d u \otimes d\left(\partial_{t} u\right)$.

Hence, it is of great importance to study the well-posedness issue of (1.24). For this model, by applying an analogous renormalization and repeating the analysis in Sect. 2 below, we see that the renormalization constant is time-independent (as in the parabolic model [8,24]). In particular, the regularity depicted in Proposition 1.1 for the relevant imaginary Gaussian chaos $\Theta$ will be time-independent in this case, implying that there exists an infinite number of threshold values for $\beta^{2}$ as in the parabolic case. By drawing an analogy to the $\Phi_{d}^{3}$-model (see [24]), we see that the $\Phi_{3}^{3}$-model corresponds to $\beta^{2}=2 \pi$. In a recent preprint [36], we establish almost

\footnotetext{
8 This is the so-called "canonical" stochastic quantization equation. See [41].

9 The Gibbs measure $\mu$ obviously requires a proper renormalization on $\mathbb{T}^{2}$.
} 
sure global well-posedness of (a renormalized version of) (1.24) and invariance of the Gibbs measure for $0<\beta^{2}<2 \pi$.

In view of a recent work [20], as for the stochastic nonlinear wave equation on $\mathbb{T}^{3}$ with a quadratic nonlinearity, we expect that it would require a significant effort (such as establishing multilinear smoothing and introducing appropriate paracontrolled operators) to reach the first non-trivial threshold $\beta^{2}=2 \pi$ for the hyperbolic sine-Gordon model (1.24). ${ }^{10}$

Remark 1.6 Albeverio et al. [1] considered the following stochastic nonlinear wave equation:

$$
\partial_{t}^{2} u+(1-\Delta) u+f(u)=\xi
$$

with zero initial data, where $f$ is assumed to be smooth and bounded. Working within the framework of Colombeau generalized functions, they showed that the solutions $u_{N}$ to (1.25) with regularized noises tend to the solution $u$ to the linear stochastic wave equation (1.22). It is, however, not clear what the meaning of the solutions constructed in [1] and how they relate to the construction in this paper. Note that in our triviality result (Proposition 1.4), we establish the convergence in the natural space $C\left([0, T] ; H^{-\varepsilon}\left(\mathbb{T}^{2}\right)\right)$. See also the comments in [8,24] on the work [2] in the parabolic setting.

Notations Before proceeding further, we introduce some notations here. We set

$$
e_{n}(x) \stackrel{\text { def }}{=} \frac{1}{2 \pi} e^{i n \cdot x}, \quad n \in \mathbb{Z}^{2},
$$

for the orthonormal Fourier basis in $L^{2}\left(\mathbb{T}^{2}\right)$. Given $s \in \mathbb{R}$, we define the Bessel potential $\langle\nabla\rangle^{s}$ of order $-s$ as a Fourier multiplier operator given by

$$
\langle\nabla\rangle^{s} f=\mathcal{F}^{-1}\left(\langle n\rangle^{s} \widehat{f}(n)\right),
$$

where $\mathcal{F}^{-1}$ denotes the inverse Fourier transform and $\langle\cdot\rangle=\left(1+|\cdot|^{2}\right)^{\frac{1}{2}}$. Then, we define the Sobolev space $H^{s}\left(\mathbb{T}^{2}\right)$ by the norm:

$$
\|f\|_{H^{s}\left(\mathbb{T}^{2}\right)}=\left\|\langle\nabla\rangle^{s} f\right\|_{L^{2}}=\left\|\langle n\rangle^{s} \widehat{f}(n)\right\|_{\ell^{2}\left(\mathbb{Z}^{2}\right)} .
$$

We also set

$$
\mathcal{H}^{s}\left(\mathbb{T}^{2}\right) \stackrel{\text { def }}{=} H^{s}\left(\mathbb{T}^{2}\right) \times H^{s-1}\left(\mathbb{T}^{2}\right) .
$$

We use short-hand notations such as $C_{T} H_{x}^{s}=C\left([0, T] ; H^{s}\left(\mathbb{T}^{2}\right)\right)$ and $L_{\omega}^{p}=L^{p}(\Omega)$.

For $A, B>0$, we use $A \lesssim B$ to mean that there exists $C>0$ such that $A \leq C B$. By $A \sim B$, we mean that $A \lesssim B$ and $B \lesssim A$. We also use a subscript to denote dependence on an external parameter; for example, $A \lesssim_{\alpha} B$ means $A \leq C(\alpha) B$,

$\overline{10}$ We point that the hyperbolic $\Phi_{3}^{3}$-model treated in [20] is much harder than the parabolic $\Phi_{3}^{3}$-model studied in [12], where a standard application of the Da Prato-Debussche trick suffices. Due to the nonpolynomial nature of the problem, we expect the hyperbolic sine-Gordon model (1.24) with $\beta^{2}=2 \pi$ to be even harder than the hyperbolic $\Phi_{3}^{3}$-model. 
where the constant $C(\alpha)>0$ depends on a parameter $\alpha$. Given two functions $f$ and $g$ on $\mathbb{T}^{2}$, we write

$$
f \approx g
$$

if there exist some constants $c_{1}, c_{2} \in \mathbb{R}$ such that $f(x)+c_{1} \leq g(x) \leq f(x)+c_{2}$ for any $x \in \mathbb{T}^{2} \backslash\{0\} \cong[-\pi, \pi)^{2} \backslash\{0\}$. Given $A, B \geq 0$, we also set $A \vee B=\max (A, B)$ and $A \wedge B=\min (A, B)$.

\section{On the imaginary Gaussian multiplicative chaos}

In this section, we study the regularity and convergence properties of the imaginary complex Gaussian chaos $\Theta_{N}=: e^{i \beta \Psi_{N}}$ : defined in (1.16) and present a proof of Proposition 1.1. As pointed out in the introduction, the main difficulty arises in that the processes $\Theta_{N}$ do not belong to any Wiener chaos of finite order. This forces us to estimate all the higher moments by hand. As in [24], the main ingredient for proving Proposition 1.1 is the bound (2.15) in Lemma 2.5, exhibiting a certain cancellation property. This allows us to simplify the expressions of the moments.

\subsection{Preliminary results}

We first recall the Poisson summation formula (see [17, Theorem 3.2.8]). In our context, it reads as follows. Let $f \in L^{1}\left(\mathbb{R}^{d}\right)$ such that (i) $|f(x)| \leq\langle x\rangle^{-d-\delta}$ for some $\delta>0$ and any $x \in \mathbb{R}^{d}$ and (ii) $\sum_{n \in \mathbb{Z}^{d}}|\widehat{f}(n)|<\infty$, where $\widehat{f}$ denotes the Fourier transform of $f$ on $\mathbb{R}^{d}$ defined by

$$
\widehat{f}(\xi)=\frac{1}{(2 \pi)^{\frac{d}{2}}} \int_{\mathbb{R}^{d}} f(x) e^{-i \xi \cdot x} d x .
$$

Then, we have

$$
\sum_{n \in \mathbb{Z}^{d}} \widehat{f}(n) e_{n}(x)=\sum_{m \in \mathbb{Z}^{d}} f(x+2 \pi m)
$$

for any $x \in \mathbb{R}^{d}$.

We also recall the following calculus lemma from [23, Lemma 3.2].

Lemma 2.1 There exists $C>0$ such that

$$
\left|\sum_{\substack{n \in \mathbb{Z}^{2} \\|n| \leq R}} \frac{1}{a+|n|^{2}}-\pi \log \left(1+\frac{R^{2}}{a}\right)\right| \leq \frac{C}{\sqrt{a}} \min \left(1, \frac{R}{\sqrt{a}}\right)
$$

for any $a, R \geq 1$. 
In $[19,20,39]$, the analysis of stochastic objects was carried out on the Fourier side. It turns out that for our problem, it is more convenient to work on the physical side. For this reason, we recall several facts from harmonic analysis. For $d \in \mathbb{N}$ and $\alpha>0$, we denote by $\langle\nabla\rangle^{-\alpha}=(1-\Delta)^{-\frac{\alpha}{2}}$ the Bessel potential of order $\alpha$. This operator on $\mathbb{T}^{d}$ is given by a convolution with the following distribution:

$$
J_{\alpha}(x) \stackrel{\text { def }}{=} \lim _{N \rightarrow \infty} \frac{1}{2 \pi} \sum_{n \in \mathbb{Z}^{d}} \frac{\chi_{N}(n)}{\langle n\rangle^{\alpha}} e_{n}(x)
$$

where $\chi_{N}$ is the smooth cutoff function onto the frequencies $\{|n| \lesssim N\}$ defined in (1.7). It is then known that for $0<\alpha<d$, the distribution $J_{\alpha}$ behaves like $|x|^{-d+\alpha}$ modulo an additive smooth function on $\mathbb{T}^{d}$. More precisely, we have the following lemma.

Lemma 2.2 Let $0<\alpha<d$. Then, the function $J_{\alpha}$ in (2.2) is smooth away from the origin and integrable on $\mathbb{T}^{d}$. Furthermore, there exist a constant $c_{\alpha, d}$ and a smooth function $R$ on $\mathbb{T}^{d}$ such that

$$
J_{\alpha}(x)=c_{\alpha, d}|x|^{\alpha-d}+R(x)
$$

for all $x \in \mathbb{T}^{d} \backslash\{0\} \cong[-\pi, \pi)^{d} \backslash\{0\}$.

Proof Let $K_{\alpha}$ be the convolution kernel for the Bessel potential on $\mathbb{R}^{d}$. Then, it follows from [18, Proposition 1.2.5] that $K_{\alpha}$ is a smooth function on $\mathbb{R}^{d} \backslash\{0\}$ and decays exponentially as $|x| \rightarrow \infty$. Moreover, the following asymptotic behavior holds:

$$
K_{\alpha}(x)=c_{\alpha, d}|x|^{\alpha-d}+o(1)
$$

as $x \rightarrow 0$. See $(4,2)$ in [3]. Then, this lemma follows from applying the Poisson summation formula (2.1) with a frequency truncation $\mathbf{P}_{N}$ and then taking $N \rightarrow \infty$ as in [17, Example 3.2.10]. See also [5].

Next, we study the Green's function for $1-\Delta$. Recall from [18, Proposition 1.2.5] that the Green's function $G_{\mathbb{R}^{2}}$ for $1-\Delta$ on $\mathbb{R}^{2}$ is a smooth function on $\mathbb{R}^{2} \backslash\{0\}$ and decays exponentially as $|x| \rightarrow \infty$. Furthermore, it satisfies

$$
G_{\mathbb{R}^{2}}(x)=-\frac{1}{2 \pi} \log |x|+o(1)
$$

as $x \rightarrow 0$. See $(4,2)$ in [3]. Now, let $G$ be the Green's function for $1-\Delta$ on $\mathbb{T}^{2}$. In view of our normalization (1.26), we then have

$$
G \stackrel{\text { def }}{=}(1-\Delta)^{-1} \delta_{0}=\frac{1}{2 \pi} \sum_{n \in \mathbb{Z}^{2}} \frac{1}{\langle n\rangle^{2}} e_{n},
$$


where the sum is to be interpreted in the limiting sense as in (2.2). Then, by applying the Poisson summation formula (2.1) (with a frequency truncation $\mathbf{P}_{N}$ and taking $N \rightarrow \infty)$ together with the asymptotics (2.3), we obtain

$$
G(x)=-\frac{1}{2 \pi} \log |x|+R(x), \quad x \in \mathbb{T}^{2} \backslash\{0\},
$$

for some smooth function $R$.

In the next lemma, we establish an analogous behavior for the frequency-truncated Green's function $\mathbf{P}_{N}^{2} G$ given by

$$
\mathbf{P}_{N}^{2} G(x)=\frac{1}{2 \pi} \sum_{n \in \mathbb{Z}^{2}} \frac{\chi_{N}^{2}(n)}{\langle n\rangle^{2}} e_{n}(x)
$$

See also Lemma 3.7 in [24].

Lemma 2.3 For any $N \in \mathbb{N}$ and $x \in \mathbb{T}^{2} \backslash\{0\}$, we have

$$
\mathbf{P}_{N}^{2} G(x) \approx-\frac{1}{2 \pi} \log \left(|x|+N^{-1}\right)
$$

where the notation $\approx$ is as in (1.27).

Proof Fix $x \in \mathbb{T}^{2} \backslash\{0\} \cong[-\pi, \pi)^{2} \backslash\{0\}$. We separately consider the cases $|x| \lesssim N^{-1}$ and $|x| \gtrsim N^{-1}$.

- Case 1 We first consider the case $|x| \lesssim N^{-1}$. By the mean value theorem, we have

$$
\left|\mathbf{P}_{N}^{2} G(x)-\mathbf{P}_{N}^{2} G(0)\right| \sim\left|\sum_{n \in \mathbb{Z}^{2}} \frac{\chi_{N}^{2}(n)}{\langle n\rangle^{2}}\left(e_{n}(x)-e_{n}(0)\right)\right| \lesssim \sum_{|n| \lesssim N} \frac{|x|}{\langle n\rangle} \lesssim N|x| \lesssim 1
$$

On the other hand, from Lemma 2.1, we have

$$
\mathbf{P}_{N}^{2} G(0)=\frac{1}{4 \pi^{2}} \sum_{n \in \mathbb{Z}^{2}} \frac{\chi_{N}^{2}(n)}{\langle n\rangle^{2}}=\frac{1}{2 \pi} \log N+O(1)
$$

as $N \rightarrow \infty$. Hence, from (2.7) and (2.8), we obtain

$$
\mathbf{P}_{N}^{2} G(x) \approx-\frac{1}{2 \pi} \log N^{-1} \approx-\frac{1}{2 \pi} \log \left(|x|+N^{-1}\right)
$$

in this case.

- Case 2 Next, we consider the case $|x| \gtrsim N^{-1}$. Let $\rho \in C^{\infty}\left(\mathbb{R}^{2}\right)$ with a compact support in $\mathbb{T}^{2} \cong[-\pi, \pi)^{2}$ with $\int \rho(y) d y=1$. Given $M \geq N$, let $\rho_{M}$ denote a 
mollification kernel of scale $M^{-1}$ given by $\rho_{M}(x)=M^{2} \rho(M x)$. Then, since $G$ and $\mathbf{P}_{N}^{2} G$ are smooth away from the origin, we have

$$
\mathbf{P}_{N}^{2} G(x)-G(x)=\lim _{M \rightarrow \infty} \rho_{M} *\left[\left(\mathbf{P}_{N}^{2}-1\right) G\right](x) .
$$

Then, by the Poisson summation formula (2.1), we write

$$
\begin{aligned}
\rho_{M} *\left[\left(\mathbf{P}_{N}^{2}-1\right) G\right](x) & =\frac{1}{2 \pi} \sum_{n \in \mathbb{Z}^{2}} \widehat{\rho}_{M}(n) \frac{\chi_{N}^{2}(n)-1}{\langle n\rangle^{2}} e_{n}(x) \\
& =\frac{1}{2 \pi} \sum_{m \in \mathbb{Z}^{2}} f_{N, M}(x+2 \pi m),
\end{aligned}
$$

where $f_{N, M} \in \mathcal{S}\left(\mathbb{R}^{2}\right)$ is defined by its Fourier transform:

$$
\widehat{f}_{N, M}(\xi)=\widehat{\rho}_{M}(\xi)\left(\chi_{N}^{2}(\xi)-1\right)\langle\xi\rangle^{-2} \in \mathcal{S}\left(\mathbb{R}^{2}\right) .
$$

By applying integrations by parts with the properties of $\chi_{N}$ and $\rho_{M}$, in particular, the fact that the integrand is essentially ${ }^{11}$ supported on $\{N \lesssim|\xi| \lesssim M\}$, we obtain

$$
\begin{aligned}
\left|f_{N, M}(x+2 \pi m)\right| & =\frac{1}{2 \pi}\left|\int_{\mathbb{R}^{2}} \widehat{f}_{N, M}(\xi) e^{i \xi \cdot(x+2 \pi m)} d \xi\right| \\
& \sim|x+2 \pi m|^{-2 k}\left|\int_{\mathbb{R}^{2}} \Delta_{\xi}^{k}\left(\widehat{\rho}_{M}(\xi)\left(\chi_{N}^{2}(\xi)-1\right)\langle\xi\rangle^{-2}\right) e^{i \xi \cdot(x+2 \pi m)} d \xi\right| \\
& \lesssim N^{-2 k}|x+2 \pi m|^{-2 k}
\end{aligned}
$$

for any $k \in \mathbb{N}$, uniformly in $M \geq N \geq 1$. When a derivative hits the second factor $\chi_{N}^{2}(\xi)-1$, we used the fact that the support in that case is essentially contained in $\{|\xi| \sim N\}$. When no derivative hits the second factor $\chi_{N}^{2}(\xi)-1$ or the third factor $\langle\xi\rangle^{-2}$, namely, when all the derivatives hit the first factor $\widehat{\rho}_{M}(\xi)=\widehat{\rho}\left(M^{-1} \xi\right)$, we used the following bound:

$$
M^{-2 k} \log \frac{M}{N}=N^{-2 k}\left(\frac{N}{M}\right)^{2 k} \log \frac{M}{N} \lesssim N^{-2 k} .
$$

By choosing $k>1$, we perform the summation over $m \in \mathbb{Z}^{2}$ and take the limit $M \rightarrow \infty$ in (2.10). From (2.9), (2.10), and (2.11), we obtain

$$
\begin{aligned}
\left|\mathbf{P}_{N}^{2} G(x)-G(x)\right| & \lesssim N^{-2 k}|x|^{-2 k}+\sum_{m \neq 0} N^{-2 k}|m|^{-2 k} \lesssim N^{-2 k}\left(|x|^{-2 k}+1\right) \\
& \lesssim(N|x|)^{-2 k} \lesssim 1,
\end{aligned}
$$

\footnotetext{
11 Namely, thanks to the fast decay of $\widehat{\rho}_{M}(\xi)$, the contribution to the integral in the second line of (2.11) from $\{|\xi| \gg M\}$ can be easily bounded by $M^{-2 k}$ for any $k \in \mathbb{N}$.
} 
under our assumption $|x| \gtrsim N^{-1}$, where we used that $|x+2 \pi m| \gtrsim|m|$ for $x \in \mathbb{T}^{2} \cong$ $[-\pi, \pi)^{2}$ and $m \neq 0$. Finally, from (2.5) and (2.12), we obtain

$$
\mathbf{P}_{N}^{2} G(x) \approx G(x) \approx-\frac{1}{2 \pi} \log |x| \approx-\frac{1}{2 \pi} \log \left(|x|+N^{-1}\right) .
$$

This completes the proof of Lemma 2.3.

Remark 2.4 Let $N_{2} \geq N_{1} \geq 1$. Then, by slightly modifying the proof of Lemma 2.3 (namely, replacing $\chi_{N}^{2}$ by $\chi_{N_{1}} \chi_{N_{2}}$ ), we have

$$
\mathbf{P}_{N_{1}} \mathbf{P}_{N_{2}} G(x) \approx-\frac{1}{2 \pi} \log \left(|x|+N_{1}^{-1}\right) .
$$

Similarly, we have

$$
\left|\mathbf{P}_{N_{j}}^{2} G(x)-\mathbf{P}_{N_{1}} \mathbf{P}_{N_{2}} G(x)\right| \lesssim\left(1 \vee-\log \left(|x|+N_{2}^{-1}\right)\right) \wedge\left(N_{1}^{-1}|x|^{-1}\right)
$$

for $j=1,2$. We point out that the second bound by $N_{1}^{-1}|x|^{-1}$ in (2.14) follows from the computation up to (2.12) in Case 2 of the proof of Lemma 2.3 (without dividing the argument, depending on the size of $|x|)$.

The next lemma plays a crucial role in proving Proposition 1.1, allowing us to reduce the product of $p(2 p-1)$ factors to that of $p$ factors.

Lemma 2.5 Let $\lambda>0$ and $p \in \mathbb{N}$. Given $j \in\{1, \ldots, 2 p\}$, we set $\varepsilon_{j}=1$ if $j$ is even and $\varepsilon_{j}=-1$ if $j$ is odd. Let $S_{p}$ be the set of all permutations of the set $\{1,2, \ldots, p\}$. Then, the following estimate holds:

$$
\prod_{1 \leq j<k \leq 2 p}\left(\left|y_{j}-y_{k}\right|+N^{-1}\right)^{\varepsilon_{j} \varepsilon_{k} \lambda} \lesssim \max _{\tau \in S_{p}} \prod_{1 \leq j \leq p}\left(\left|y_{2 j}-y_{2 \tau(j)-1}\right|+N^{-1}\right)^{-\lambda}
$$

for any set $\left\{y_{j}\right\}_{j=1, \ldots, 2 p}$ of $2 p$ points in $\mathbb{T}^{2}$ and any $N \in \mathbb{N}$.

In [24], Hairer and Shen established an analogous result for a more general class of "potential" function $\mathcal{J}_{N}(x-y)$ than $\left(|x-y|+N^{-1}\right)^{\lambda}$ considered above. They exploited sophisticated multi-scale analysis and charge cancellation. In particular, Lemma 2.5 follows from Proposition 3.5 in [24]. Nonetheless, we decided to include a proof since the relevant computation is elementary and concise in our concrete setting. In the proof below, the charge cancellation due to the "dipole" formation appears in (2.19) and (2.20). We also mention the work [13] where an exact identity (see (3.13) in [13]) was used to study a similar problem.

Proof We prove (2.15) by induction on $p$. When $p=1,(2.15)$ holds with an equality. Now, let us assume that (2.15) holds for some $p \in \mathbb{N}$ and fix a set $\left\{y_{j}\right\}_{j=1, \ldots, 2(p+1)}$ of $2(p+1)$ points in $\mathbb{T}^{2}$. By defining the sets $A_{p}^{+}=\{j=1, \ldots, 2 p, j$ even $\}$ and $A_{p}^{-}=\{k=1, \ldots, 2 p, k$ odd $\}$, we set

$$
A_{p}^{\sigma_{1} \sigma_{2}}=\left\{(j, k) \in\{1, \ldots, 2 p\}: j<k, j \in A_{p}^{\sigma_{1}}, k \in A_{p}^{\sigma_{2}}\right\}
$$


for $\sigma_{1}, \sigma_{2} \in\{+,-\}$. With a slight abuse of notation, we identify + and - with +1 and -1 in the following.

We denote by $\Pi_{p}$ the left-hand side of (2.15). Then, we have

$$
\Pi_{p+1}=\frac{\prod_{\substack{\sigma_{1}, \sigma_{2} \in\{+,-\} \\ \sigma_{1} \sigma_{2}=1}} \prod_{(j, k) \in A_{p+1}^{\sigma_{1} \sigma_{2}}}\left(\left|y_{j}-y_{k}\right|+N^{-1}\right)^{\lambda}}{\prod_{\substack{\sigma_{1}, \sigma_{2} \in\{+,-\} \\ \sigma_{1} \sigma_{2}=-1}} \prod_{(j, k) \in A_{p+1}^{\sigma_{1} \sigma_{2}}}\left(\left|y_{j}-y_{k}\right|+N^{-1}\right)^{\lambda}} .
$$

Namely, the numerator contains all the factors with the same parity for $j$ and $k$, while the denominator contains all the factors with the opposite parities for $j$ and $k$. Now, choose $j_{0} \in A_{p+1}^{+}$and $k_{0} \in A_{p+1}^{-}$such that

$$
\left|y_{j_{0}}-y_{k_{0}}\right|=\min \left\{\left|y_{j}-y_{k}\right| ; j \in A_{p+1}^{+}, k \in A_{p+1}^{-}\right\} .
$$

It follows from its definition given by the left-hand side of (2.15) (with $p$ replaced by $p+1)$ that $\Pi_{p+1}$ is invariant under permutations of $\{1, \ldots, 2 p+2\}$ that do not mix even and odd integers (i.e. not mixing the + and - charges). Thus, without loss of generality, we assume that $\left(j_{0}, k_{0}\right)=(2 p+1,2 p+2)$.

By the inductive hypothesis we have

$$
\Pi_{p} \lesssim \max _{\tau \in S_{p}} \prod_{1 \leq j \leq p}\left(\left|y_{2 j}-y_{2 \tau(j)-1}\right|+N^{-1}\right)^{-\lambda} .
$$

In view of (2.15), it suffices to prove the following bound:

$$
\frac{\Pi_{p+1}}{\Pi_{p}} \lesssim\left(\left|y_{2 p+1}-y_{2 p+2}\right|+N^{-1}\right)^{-\lambda}
$$

uniformly in $N$. Note that the left-hand side of (2.17) contains only the factors involving $y_{2 p+1}$ or $y_{2 p+2}$. Hence, we have

$$
\begin{aligned}
& \frac{\Pi_{p+1}}{\Pi_{p}}\left(\left|y_{2 p+1}-y_{2 p+2}\right|+N^{-1}\right)^{\lambda} \\
& \quad=\prod_{1 \leq j \leq p}\left(\frac{\left|y_{2 j-1}-y_{2 p+1}\right|+N^{-1}}{\left|y_{2 j-1}-y_{2 p+2}\right|+N^{-1}}\right)^{\lambda}\left(\frac{\left|y_{2 j}-y_{2 p+2}\right|+N^{-1}}{\left|y_{2 j}-y_{2 p+1}\right|+N^{-1}}\right)^{\lambda} .
\end{aligned}
$$

On the other hand, by the triangle inequality and (2.16), we have

$$
\max _{1 \leq j \leq p} \frac{\left|y_{2 j-1}-y_{2 p+1}\right|+N^{-1}}{\left|y_{2 j-1}-y_{2 p+2}\right|+N^{-1}} \leq 1+\max _{1 \leq j \leq p} \frac{\left|y_{2 p+1}-y_{2 p+2}\right|}{\left|y_{2 j-1}-y_{2 p+2}\right|+N^{-1}} \lesssim 1,
$$

uniformly in $N$. Similarly, we have

$$
\max _{1 \leq j \leq p} \frac{\left|y_{2 j}-y_{2 p+2}\right|+N^{-1}}{\left|y_{2 j}-y_{2 p+1}\right|+N^{-1}} \lesssim 1,
$$


uniformly in $N$. Therefore, the desired estimate (2.17) follows from (2.18), (2.19), and (2.20).

\subsection{Estimates on the stochastic objects}

In this subsection, we present a proof of Proposition 1.1 on the imaginary complex Gaussian chaos $\Theta_{N}=: e^{i \beta \Psi_{N}}$ : defined in (1.16).

We first recall the following lemma from [19, Proposition 2.1] on the regularity of the truncated stochastic convolution $\Psi_{N}$. See also [20, Lemma 3.1].

Lemma 2.6 Given any $T, \varepsilon>0$ and finite $p \geq 1,\left\{\Psi_{N}\right\}_{N \in \mathbb{N}}$ is a Cauchy sequence in $L^{p}\left(\Omega ; C\left([0, T] ; W^{-\varepsilon, \infty}\left(\mathbb{T}^{2}\right)\right)\right)$, converging to some limit $\Psi$ in $L^{p}(\Omega ; C([0, T] ;$ $\left.\left.W^{-\varepsilon, \infty}\left(\mathbb{T}^{2}\right)\right)\right)$. Moreover, $\Psi_{N}$ converges almost surely to the same limit $\Psi \in$ $C\left([0, T] ; W^{-\varepsilon, \infty}\left(\mathbb{T}^{2}\right)\right)$.

It is easy to show that the claim in Lemma 2.6 fails when $\varepsilon=0$. Thus, as $N \rightarrow$ $\infty, \Psi_{N}$ becomes singular, necessitating a proper renormalization procedure for $\Theta_{N}$ defined in (1.16).

As mentioned above, we will work on the physical space (rather than on the Fourier side as in [19,20,39]). For this purpose, we first study the covariance function for $\Psi_{N}$. Let

$$
\Gamma_{N}(t, x-y) \stackrel{\text { def }}{=} \mathbb{E}\left[\Psi_{N}(t, x) \Psi_{N}(t, y)\right] .
$$

Lemma 2.7 Given $N \in \mathbb{N}$, let $\Gamma_{N}$ be as in (2.21). Then, we have

$$
\Gamma_{N}(t, x-y)=\frac{1}{2 \pi} \sum_{n \in \mathbb{Z}^{2}} \chi_{N}^{2}(n)\left\{\frac{t}{2\langle n\rangle^{2}}-\frac{\sin (2 t\langle n\rangle)}{4\langle n\rangle^{3}}\right\} e_{n}(x-y)
$$

In particular, we have

$$
\Gamma_{N}(t, x-y) \approx-\frac{t}{4 \pi} \log \left(|x-y|+N^{-1}\right)
$$

for any $t \in \mathbb{R}$.

Proof The identity (2.22) follows from a straightforward computation, using (1.10) and (1.11) with (1.26). See (1.8) and (2.8) in [19] for analogous computations.

From (2.6) and (2.22), we have

$$
\Gamma_{N}(t, x) \approx \frac{t}{2} \mathbf{P}_{N}^{2} G(x)
$$

Then, (2.23) follows from Lemma 2.3.

By setting

$$
\Gamma(t, x-y) \stackrel{\text { def }}{=} \mathbb{E}[\Psi(t, x) \Psi(t, y)],
$$


we then formally have

$$
\Gamma(t, x-y)=\frac{1}{2 \pi} \sum_{n \in \mathbb{Z}^{2}}\left\{\frac{t}{2\langle n\rangle^{2}}-\frac{\sin (2 t\langle n\rangle)}{4\langle n\rangle^{3}}\right\} e_{n}(x-y),
$$

where the sum is to be interpreted in the limiting sense as in (2.2). By comparing (2.4) and (2.24), we have $\Gamma(t, x) \approx \frac{t}{2} G(x)$. Similarly, we have

$$
\mathbf{P}_{N_{1}} \mathbf{P}_{N_{2}} \Gamma(t, x) \approx \frac{t}{2} \mathbf{P}_{N_{1}} \mathbf{P}_{N_{2}} G(x)
$$

In particular, from (2.13) in Remark 2.4, we obtain

$$
\mathbf{P}_{N_{1}} \mathbf{P}_{N_{2}} \Gamma(t, x) \approx-\frac{t}{4 \pi} \log \left(|x|+N_{1}^{-1}\right)
$$

for $N_{2} \geq N_{1} \geq 1$.

We are now ready to present a proof of Proposition 1.1.

Proof of Proposition 1.1 Fix $\beta \in \mathbb{R}$ and $T>0$ such that $\beta^{2} T<8 \pi$. Also let $p \in \mathbb{N}$, finite $q \geq 1$, and $\alpha>\frac{\beta^{2} T}{8 \pi}$. Without loss of generality, we assume $\alpha<2$ in the following.

Fix small $\delta>0$. Then, by Sobolev's inequality (in $x$ ) followed by Minkowski's integral inequality, we have

$$
\left\|\Theta_{N}\right\|_{L_{\omega}^{2 p} L_{T}^{q} W_{x}^{-\alpha, \infty}} \lesssim\left\|\langle\nabla\rangle^{\delta-\alpha} \Theta_{N}\right\|_{L_{\omega}^{2 p} L_{T}^{q} L_{x}^{r_{\delta}}} \lesssim\|\|\langle\nabla\rangle^{\delta-\alpha} \Theta_{N}(t, x)\left\|_{L_{\omega}^{2 p}}\right\|_{L_{T}^{q} L_{x}^{r_{\delta}}}
$$

for some large but finite $r_{\delta}$, provided that $2 p \geq \max \left(q, r_{\delta}\right)$.

Fix $t \in[0, T]$ and $x \in \mathbb{T}^{2}$. Recalling $\langle\nabla\rangle^{\delta-\alpha} f=J_{\alpha-\delta} * f$, where $J_{\alpha}$ is as in (2.2), it follows from (1.16) that

$$
\begin{aligned}
\mathbb{E}\left[\left|\langle\nabla\rangle^{\delta-\alpha} \Theta_{N}(t, x)\right|^{2 p}\right]= & e^{p \beta^{2} \sigma_{N}(t)} \mathbb{E}\left[\left|\int_{\mathbb{T}^{2}} J_{\alpha-\delta}(x-y) e^{i \beta \Psi_{N}(t, y)} d y\right|^{2 p}\right] \\
= & e^{p \beta^{2} \sigma_{N}(t)} \int_{\left(\mathbb{T}^{2}\right)^{2 p}} \mathbb{E}\left[e^{i \beta \sum_{j=1}^{p}\left(\Psi_{N}\left(t, y_{2 j}\right)-\Psi_{N}\left(t, y_{2 j}-1\right)\right)}\right] \\
& \times \prod_{k=1}^{2 p} J_{\alpha-\delta}\left(x-y_{k}\right) d \vec{y}
\end{aligned}
$$

where $d \vec{y} \stackrel{\text { def }}{=} d y_{1} \cdots d y_{2} p$. Noting that $\sum_{j=1}^{p}\left(\Psi_{N}\left(t, y_{2 j}\right)-\Psi_{N}\left(t, y_{2 j-1}\right)\right)$ is a meanzero Gaussian random variable, the explicit formula for the characteristic function of a Gaussian random variable yields 


$$
\mathbb{E}\left[e^{i \beta \sum_{j=1}^{p}\left(\Psi_{N}\left(t, y_{2 j}\right)-\Psi_{N}\left(t, y_{2 j-1}\right)\right)}\right]=e^{-\frac{\beta^{2}}{2} \mathbb{E}\left[\left|\sum_{j=1}^{p}\left(\Psi_{N}\left(t, y_{2 j}\right)-\Psi_{N}\left(t, y_{2 j-1}\right)\right)\right|^{2}\right]}
$$

Let $\left\{\varepsilon_{j}\right\}_{j=1, \ldots, 2 p}$ be as in Lemma 2.5. Then, we can rewrite the expectation in the exponent on the right-hand side of (2.29) as

$$
\mathbb{E}\left[\left|\sum_{j=1}^{2 p} \varepsilon_{j} \Psi_{N}\left(t, y_{j}\right)\right|^{2}\right]=\sum_{j, k=1}^{2 p} \varepsilon_{j} \varepsilon_{k} \Gamma_{N}\left(t, y_{j}-y_{k}\right)
$$

where $\Gamma_{N}$ is the covariance function defined in (2.21). From the definition (1.12), we have $\Gamma_{N}(t, 0)=\sigma_{N}(t)$. Hence, we obtain

$$
e^{-\frac{\beta^{2}}{2} \mathbb{E}\left[\left|\sum_{j=1}^{p}\left(\Psi_{N}\left(t, y_{2 j}\right)-\Psi_{N}\left(y_{2 j-1}, t\right)\right)\right|^{2}\right]}=e^{-p \beta^{2} \sigma_{N}(t)} e^{-\beta^{2} \sum_{1 \leq j<k \leq 2 p} \varepsilon_{j} \varepsilon_{k} \Gamma_{N}\left(t, y_{j}-y_{k}\right)} .
$$

Then, from (2.29), (2.30), the two-sided bound (2.23) in Lemmas 2.7 and 2.5, we obtain

$$
\begin{aligned}
& e^{p \beta^{2} \sigma_{N}(t)} \mathbb{E}\left[e^{i \beta \sum_{j=1}^{p}\left(\Psi_{N}\left(t, y_{2 j}\right)-\Psi_{N}\left(t, y_{2 j}-1\right)\right)}\right] \\
& \sim \prod_{1 \leq j<k \leq 2 p}\left(\left|y_{j}-y_{k}\right|+N^{-1}\right)^{\varepsilon_{j} \varepsilon_{k} \frac{\beta^{2} t}{4 \pi}} \\
& \quad \lesssim \max _{\tau \in S_{p}} \prod_{1 \leq j \leq p}\left(\left|y_{2 j}-y_{2 \tau(j)-1}\right|+N^{-1}\right)^{-\frac{\beta^{2} t}{4 \pi}} \\
& \leq \sum_{\tau \in S_{p}} \prod_{1 \leq j \leq p}\left(\left|y_{2 j}-y_{2 \tau(j)-1}\right|+N^{-1}\right)^{-\frac{\beta^{2} t}{4 \pi}} .
\end{aligned}
$$

Finally, from (2.28) and (2.31) we obtain

$$
\begin{aligned}
& \mathbb{E}\left[\left|\langle\nabla\rangle^{\delta-\alpha} \Theta_{N}(t, x)\right|^{2 p}\right] \\
& \quad \lesssim \sum_{\tau \in S_{p}} \int_{\left(\mathbb{T}^{2}\right)^{2 p}} \prod_{1 \leq j \leq p}\left(\left|y_{2 j}-y_{2 \tau(j)-1}\right|+N^{-1}\right)^{-\frac{\beta^{2} t}{4 \pi}} \prod_{k=1}^{2 p}\left|J_{\alpha-\delta}\left(x-y_{k}\right)\right| d \vec{y} .
\end{aligned}
$$

In the following, we fix $\tau \in S_{p}$. Then, it suffices to bound each pair of integrals:

$$
\int_{\mathbb{T}^{2}} \int_{\mathbb{T}^{2}}\left(\left|y_{j}-y_{k}\right|+N^{-1}\right)^{-\frac{\beta^{2} t}{4 \pi}}\left|J_{\alpha-\delta}\left(x-y_{j}\right)\right|\left|J_{\alpha-\delta}\left(x-y_{k}\right)\right| d y_{j} d y_{k},
$$


for an even integer $j=2, \ldots, 2 p$ and $k=2 \tau\left(\frac{j}{2}\right)-1$. From Lemma 2.2 with $0<\alpha-\delta<2$, we can bound this integral by

$$
\begin{gathered}
\int_{\mathbb{T}^{2}} \int_{\mathbb{T}^{2}}\left(\left|y_{j}-y_{k}\right|+N^{-1}\right)^{-\frac{\beta^{2} t}{4 \pi}}\left|x-y_{j}\right|^{\alpha-\delta-2}\left|x-y_{k}\right|^{\alpha-\delta-2} d y_{j} d y_{k} \\
\leq \int_{\mathbb{T}^{2}} \int_{\mathbb{T}^{2}}\left|y_{j}-y_{k}\right|^{-\frac{\beta^{2} t}{4 \pi}}\left|x-y_{j}\right|^{\alpha-\delta-2}\left|x-y_{k}\right|^{\alpha-\delta-2} d y_{j} d y_{k},
\end{gathered}
$$

uniformly in $N \in \mathbb{N}$.

- Case $1\left|y_{j}-y_{k}\right| \sim\left|x-y_{k}\right| \gtrsim\left|x-y_{j}\right|$. In this case, we bound the integral in (2.33) by

$$
\begin{aligned}
\text { RHS of (2.33) } & \lesssim \int_{\mathbb{T}^{2}}\left|x-y_{k}\right|^{\alpha-\delta-2-\frac{\beta^{2} t}{4 \pi}} \int_{\left|x-y_{j}\right| \lesssim\left|x-y_{k}\right|}\left|x-y_{j}\right|^{\alpha-\delta-2} d y_{j} d y_{k} \\
& \lesssim \int_{\mathbb{T}^{2}}\left|x-y_{k}\right|^{2(\alpha-\delta)-2-\frac{\beta^{2} t}{4 \pi}} d y_{k} \lesssim 1,
\end{aligned}
$$

where we used $\frac{\beta^{2} t}{8 \pi}<\alpha-\delta$. The symmetry allows us to handle the case $\left|y_{j}-y_{k}\right| \sim$ $\left|x-y_{j}\right| \gtrsim\left|x-y_{k}\right|$.

- Case $2\left|x-y_{j}\right| \sim\left|x-y_{k}\right| \gtrsim\left|y_{j}-y_{k}\right|$. In this case, we bound the integral in (2.33) by

$$
\begin{aligned}
\text { RHS of (2.33) } & \lesssim \int_{\mathbb{T}^{2}}\left|x-y_{k}\right|^{2(\alpha-\delta)-4} \int_{\left|y_{j}-y_{k}\right| \lesssim\left|x-y_{k}\right|}\left|y_{j}-y_{k}\right|^{-\frac{\beta^{2} t}{4 \pi}} d y_{j} d y_{k} \\
& \lesssim \int_{\mathbb{T}^{2}}\left|x-y_{k}\right|^{2(\alpha-\delta)-2-\frac{\beta^{2} t}{4 \pi}} d y_{k} \lesssim 1
\end{aligned}
$$

since $\frac{\beta^{2} t}{8 \pi}<\min (\alpha-\delta, 1)$.

Putting together (2.32) and the estimates on (2.33), we obtain

$$
\mathbb{E}\left[\left|\langle\nabla\rangle^{\delta-\alpha} \Theta_{N}(t, x)\right|^{2 p}\right] \lesssim 1
$$

uniformly in $t \in[0, T], x \in \mathbb{T}^{2}$, and $N \in \mathbb{N}$. Therefore, we conclude from (2.27) and (2.34) that

$$
\left\|\Theta_{N}\right\|_{L_{\omega}^{2 p} L_{T}^{q} W_{x}^{-\alpha, \infty}} \lesssim T^{\frac{1}{q}}
$$

uniformly in $N \in \mathbb{N}$. 
Next, we establish convergence of $\Theta_{N}$. Let $N_{2} \geq N_{1} \geq 1$. By repeating the computation as above when $p=1$ with $\Theta_{N_{1}}-\Theta_{N_{2}}$ in place of $\Theta_{N}$, we have

$$
\begin{aligned}
\mathbb{E} & {\left[\left|\langle\nabla\rangle^{\delta-\alpha}\left(\Theta_{N_{1}}(t, x)-\Theta_{N_{2}}(t, x)\right)\right|^{2}\right] } \\
= & \int_{\mathbb{T}^{2}} \int_{\mathbb{T}^{2}} J_{\alpha-\delta}(x-y) J_{\alpha-\delta}(x-z) \\
& \times \mathbb{E}\left[\left(e^{\frac{\beta^{2}}{2} \sigma_{N_{1}}(t)} e^{i \beta \Psi_{N_{1}}(t, y)}-e^{\frac{\beta^{2}}{2} \sigma_{N_{2}}(t)} e^{i \beta \Psi_{N_{2}}(t, y)}\right)\right. \\
& \left.\times\left(e^{\frac{\beta^{2}}{2} \sigma_{N_{1}}(t)} e^{-i \beta \Psi_{N_{1}}(t, z)}-e^{\frac{\beta^{2}}{2} \sigma_{N_{2}}(t)} e^{-i \beta \Psi_{N_{2}}(t, z)}\right)\right] d y d z \\
= & \sum_{j=1}^{2} \int_{\mathbb{T}^{2}} \int_{\mathbb{T}^{2}} J_{\alpha-\delta}(x-y) J_{\alpha-\delta}(x-z)\left(e^{\beta^{2} \Gamma_{N_{j}}(t, y-z)}-e^{\beta^{2} \mathbf{P}_{N_{1}} \mathbf{P}_{N_{2}} \Gamma(t, y-z)}\right) d y d z,
\end{aligned}
$$

where we used $\mathbf{P}_{N_{1}} \mathbf{P}_{N_{2}} \Gamma(t, y-z)=\mathbb{E}\left[\Psi_{N_{1}}(t, y) \Psi_{N_{2}}(t, z)\right]$. By the mean value theorem and the bounds (2.23) in Lemma 2.7 and (2.26), we have

$$
\begin{aligned}
\left|e^{\beta^{2} \Gamma_{N_{j}}(t, x)}-e^{\beta^{2} \mathbf{P}_{N_{1}} \mathbf{P}_{N_{2}} \Gamma(t, x)}\right| \\
=\mid\left(\mathbf{P}_{N_{j}}^{2} \Gamma(t, x)-\mathbf{P}_{N_{1}} \mathbf{P}_{N_{2}} \Gamma(t, x)\right) \\
\quad \times \int_{0}^{1} \beta^{2} \exp \left[\beta^{2}\left(\tau \mathbf{P}_{N_{j}}^{2} \Gamma(t, x)+(1-\tau) \mathbf{P}_{N_{1}} \mathbf{P}_{N_{2}} \Gamma(t, x)\right)\right] d \tau \mid \\
\quad \lesssim\left(|x|+N_{2}^{-1}\right)^{-\frac{\beta^{2} t}{4 \pi}}\left|\mathbf{P}_{N_{j}}^{2} \Gamma(t, x)-\mathbf{P}_{N_{1}} \mathbf{P}_{N_{2}} \Gamma(t, x)\right| .
\end{aligned}
$$

Given $\varepsilon>0$, there exists $C_{\varepsilon}>0$ such that

$$
|\log y| \leq C_{\varepsilon} y^{-\varepsilon}
$$

for any $0<y \lesssim 1$. From (2.14) and (2.25) along with (2.37), we have

$$
\begin{aligned}
\left|\mathbf{P}_{N_{j}}^{2} \Gamma(t, x)-\mathbf{P}_{N_{1}} \mathbf{P}_{N_{2}} \Gamma(t, x)\right| & \lesssim T\left(|x|+N_{2}^{-1}\right)^{-\varepsilon} \wedge\left(N_{1}^{-1}|x|^{-1}\right) \\
& \lesssim T N_{1}^{-\varepsilon}|x|^{-2 \varepsilon}
\end{aligned}
$$

for any $t \in[0, T]$ and non-zero $x \in \mathbb{T}^{2} \cong[-\pi, \pi)^{2}$. Hence, from (2.35), (2.36), and (2.38) along with Lemma 2.2 (with $0<\alpha-\delta<2$ ), we obtain

$$
\begin{aligned}
& \mathbb{E}\left[\left|\langle\nabla\rangle^{\delta-\alpha}\left(\Theta_{N_{1}}(t, x)-\Theta_{N_{2}}(t, x)\right)\right|^{2}\right] \\
& \quad \lesssim T \int_{\mathbb{T}^{2}} \int_{\mathbb{T}^{2}}|x-y|^{\alpha-\delta-2}|x-z|^{\alpha-\delta-2} N_{1}^{-\varepsilon}|y-z|^{-2 \varepsilon-\frac{\beta^{2} t}{4 \pi}} d y d z .
\end{aligned}
$$


By taking $\varepsilon>0$ sufficiently small such that $\frac{\beta^{2} t}{8 \pi}+\varepsilon<\min (\alpha-\delta, 1)$, we can proceed as in Cases 1 and 2 above and estimate the integrals above by

$$
\mathbb{E}\left[\left|\langle\nabla\rangle^{\delta-\alpha}\left(\Theta_{N_{1}}(t, x)-\Theta_{N_{2}}(t, x)\right)\right|^{2}\right] \lesssim T N_{1}^{-\varepsilon}
$$

for any $N_{2} \geq N_{1} \geq 1, t \in[0, T]$, and $x \in \mathbb{T}^{2}$.

Given $p \geq 1$, we can interpolate the previous bound (2.39) with (2.34) and obtain

$$
\mathbb{E}\left[\left|\langle\nabla\rangle^{\delta-\alpha}\left(\Theta_{N_{1}}(t, x)-\Theta_{N_{2}}(t, x)\right)\right|^{p}\right] \leq C(T) N_{1}^{-\varepsilon}
$$

for any $N_{2} \geq N_{1} \geq 1, t \in[0, T]$, and $x \in \mathbb{T}^{2}$. Therefore, we conclude that

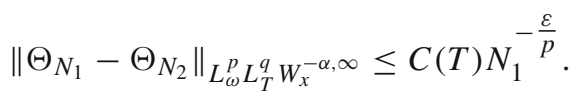

This shows that $\Theta_{N}$ is a Cauchy sequence in $L^{p}\left(\Omega ; L^{q}\left([0, T] ; W^{-\alpha, \infty}\left(\mathbb{T}^{2}\right)\right)\right)$. This completes the proof of Proposition 1.1.

\section{Proofs of the main results}

In this section, we present a proof of local well-posedness of the renormalized stochastic sine-Gordon equation (Theorem 1.2). Given the regularity and convergence properties for the imaginary Gaussian multiplicative chaos $\Theta_{N}$ (Proposition 1.1), Theorem 1.2 follows from a standard application of the Strichartz estimate (see (3.3) below) as in the work [19] on the stochastic nonlinear wave equation with a polynomial nonlinearity. For this purpose, we first go over the Strichartz estimate along with other useful lemmas in Sect. 3.1. We then present a proof of Theorem 1.2 in Sect. 3.2. We conclude this paper by establishing a triviality result (Proposition 1.4) for the (unrenormalized) stochastic sine-Gordon equation (1.1) in Sect. 3.3.

\subsection{Strichartz estimates and other useful lemmas}

We begin by recalling the Strichartz estimate for the linear wave equation on $\mathbb{T}^{2}$. Given $0<s<1$, we say that a pair $(q, r)$ of exponents (and a pair $(\widetilde{q}, \widetilde{r})$, respectively) is $s$-admissible (and dual $s$-admissible, respectively), if $1 \leq \widetilde{q} \leq 2 \leq q \leq \infty$ and $1<\tilde{r} \leq 2 \leq r<\infty$ and if they satisfy the following scaling and admissibility conditions:

$$
\frac{1}{q}+\frac{2}{r}=1-s=\frac{1}{\widetilde{q}}+\frac{2}{\widetilde{r}}-2, \quad \frac{2}{q}+\frac{1}{r} \leq \frac{1}{2}, \quad \text { and } \quad \frac{2}{\widetilde{q}}+\frac{1}{\widetilde{r}} \geq \frac{5}{2} .
$$

Then, it follows from Lemma 3.3 in [19], which studies the maximization problem for $J_{s}=\min \left(\frac{r}{r}, \frac{q}{q}\right), 0<s<1$, that given $0<s<1$, there exist an $s$-admissible pair 
$(q, r)=(q(s), r(s))$ and a dual $s$-admissible pair $(\widetilde{q}, \widetilde{r})=(\widetilde{q}(s), \widetilde{r}(s))$ such that

$$
q>2 \widetilde{q} \text { and } r>2 \widetilde{r}
$$

Let $0<s<1$. In the remaining part of this paper, we fix pairs $(q, r)$ and $(\widetilde{q}, \widetilde{r})$ as above, satisfying (3.1). Given $0<T \leq 1$, we then define the Strichartz space:

$$
X^{S}(T)=C\left([0, T] ; H^{S}\left(\mathbb{T}^{2}\right)\right) \cap C^{1}\left([0, T] ; H^{s-1}\left(\mathbb{T}^{2}\right)\right) \cap L^{q}\left([0, T] ; L^{r}\left(\mathbb{T}^{2}\right)\right)
$$

and its "dual" space:

$$
N^{S}(T)=L^{1}\left([0, T] ; H^{s-1}\left(\mathbb{T}^{2}\right)\right)+L^{\widetilde{q}}\left([0, T] ; L^{\widetilde{r}}\left(\mathbb{T}^{2}\right)\right),
$$

where $X^{s}(T)$ - and $N^{s}(T)$-norms are given by

$$
\|u\|_{X^{s}(T)}=\|u\|_{C_{T} H_{x}^{s}}+\left\|\partial_{t} u\right\|_{C_{T} H_{x}^{s-1}}+\|u\|_{L_{T}^{q} L_{x}^{r}}
$$

and

$$
\|u\|_{N^{s}(T)}=\inf \left\{\left\|u_{1}\right\|_{L_{T}^{1} H_{x}^{s-1}}+\left\|u_{2}\right\|_{L_{T}^{\widetilde{q}} L_{x}^{\tilde{r}}}: u=u_{1}+u_{2}\right\} .
$$

Then, the solution $u$ to the following linear wave equation:

$$
\left\{\begin{array}{l}
\partial_{t}^{2} u+(1-\Delta) u=f \\
\left.\left(u, \partial_{t} u\right)\right|_{t=0}=\left(u_{0}, u_{1}\right)
\end{array}\right.
$$

on $[0, T]$ satisfies the following Strichartz estimate:

$$
\|u\|_{X^{s}(T)} \lesssim\left\|\left(u_{0}, u_{1}\right)\right\|_{\mathcal{H}^{s}}+\|f\|_{N^{s}(T)},
$$

uniformly in $T \in[0,1]$. The Strichartz estimates on $\mathbb{R}^{d}$ have been studied extensively by many mathematicians. See $[16,26,30]$ in the context of the wave equation. For the Klein-Gordon equation under consideration, see [27]. Thanks to the finite speed of propagation, the estimate (3.3) on $\mathbb{T}^{2}$ follows from the corresponding estimate on $\mathbb{R}^{2}$.

Next, we recall the following product estimates. See Lemma 3.4 in [19].

Lemma 3.1 Let $0 \leq s \leq 1$.

(i) Suppose that $1<p_{j}, q_{j}, r<\infty, \frac{1}{p_{j}}+\frac{1}{q_{j}}=\frac{1}{r}, j=1,2$. Then, we have

$$
\left\|\langle\nabla\rangle^{s}(f g)\right\|_{L^{r}\left(\mathbb{T}^{d}\right)} \lesssim\left(\|f\|_{L^{p_{1}\left(\mathbb{T}^{d}\right)}}\left\|\langle\nabla\rangle^{s} g\right\|_{L^{q_{1}\left(\mathbb{T}^{d}\right)}}+\left\|\langle\nabla\rangle^{s} f\right\|_{L^{p_{2}\left(\mathbb{T}^{d}\right)}}\|g\|_{L^{q_{2}\left(\mathbb{T}^{d}\right)}}\right) .
$$

(ii) Suppose that $1<p, q, r<\infty$ satisfy the scaling condition: $\frac{1}{p}+\frac{1}{q}=\frac{1}{r}+\frac{s}{d}$.

Then, we have

$$
\left\|\langle\nabla\rangle^{-s}(f g)\right\|_{L^{r}\left(\mathbb{T}^{d}\right)} \lesssim\left\|\langle\nabla\rangle^{-s} f\right\|_{L^{p}\left(\mathbb{T}^{d}\right)}\left\|\langle\nabla\rangle^{s} g\right\|_{L^{q}\left(\mathbb{T}^{d}\right)} .
$$


Lastly, we recall the fractional chain rule from [15].

Lemma 3.2 Suppose that $F$ is a Lipschitz function with Lipschitz constant L. Then, given any $0<s<1$ and $1<p<\infty$, we have

$$
\left\||\nabla|^{s} F(u)\right\|_{L^{p}\left(\mathbb{T}^{d}\right)} \lesssim L\left\||\nabla|^{s} u\right\|_{L^{p}\left(\mathbb{T}^{d}\right)}
$$

for any $u \in C^{\infty}\left(\mathbb{T}^{d}\right)$.

The fractional chain rule on $\mathbb{R}^{d}$ was essentially proved in [9]. ${ }^{12}$ As for the estimate (3.6) on $\mathbb{T}^{d}$, see [15].

\subsection{Local well-posedness}

In this subsection, we present a proof of Theorem 1.2. For simplicity, we assume that $0<s<1$, in which case we have $\sigma=s$ in the statement of Theorem 1.2. Fix $\beta \in \mathbb{R} \backslash\{0\}$ and $\left(u_{0}, u_{1}\right) \in \mathcal{H}^{s}\left(\mathbb{T}^{2}\right)$. The main idea is to apply the Da Prato-Debussche trick. Namely, we first write the solution $u_{N}$ to (1.17) as $u_{N}=\Psi_{N}+v_{N}$, where $\Psi_{N}$ is the truncated stochastic convolution defined in (1.10). Then, the residual term $v_{N}$ satisfies (1.19). By writing (1.19) in the Duhamel formulation, we have

$$
v_{N}(t)=\partial_{t} S(t) u_{0}+S(t) u_{1}-\int_{0}^{t} S\left(t-t^{\prime}\right) \operatorname{Im}\left(\Theta_{N} e^{i \beta v_{N}}\right)\left(t^{\prime}\right) d t^{\prime},
$$

where $S(t)$ and $\Theta_{N}$ are as in (1.9) and (1.16), respectively. In the following, we use $B_{R}$ to denote the ball in $X^{s}(T)$ of radius $R>0$ centered at the origin.

Let $\Phi^{N}\left(v_{N}\right)=\Phi_{\left(u_{0}, u_{1}\right), \Theta_{N}}^{N}\left(v_{N}\right)$ denote the right-hand side of (3.7). Let $0<T \leq 1$. By the Strichartz estimate (3.3), we have

$$
\left\|\Phi^{N}\left(v_{N}\right)\right\|_{X^{s}(T)} \lesssim\left\|\left(u_{0}, u_{1}\right)\right\|_{\mathcal{H}^{s}}+\left\|\operatorname{Im}\left(\Theta_{N} e^{i \beta v_{N}}\right)\right\|_{N^{s}(T)}
$$

From (3.22) and (3.23) in [19], we have

$$
\|f\|_{L_{T}^{q_{1}} W_{x}^{\alpha, r_{1}}} \lesssim\|f\|_{X^{s}(T)} \text { and }\|f\|_{N^{s}(T)} \lesssim\|f\|_{L_{T}^{\widetilde{q}_{1}} W_{x}^{-\alpha, \widetilde{r}_{1}}},
$$

where $0<\alpha<\min (s, 1-s)$ and the pairs $\left(q_{1}, r_{1}\right)$ and $\left(\widetilde{q}_{1}, \widetilde{r}_{1}\right)$ are given by

$$
\frac{1}{q_{1}}=\frac{1-\alpha / s}{q}+\frac{\alpha / s}{\infty}, \quad \frac{1}{r_{1}}=\frac{1-\alpha / s}{r}+\frac{\alpha / s}{2}
$$

and

$$
\frac{1}{\widetilde{q}_{1}}=\frac{\alpha /(1-s)}{1}+\frac{1-\alpha /(1-s)}{\widetilde{q}}, \quad \frac{1}{\widetilde{r}_{1}}=\frac{\alpha /(1-s)}{2}+\frac{1-\alpha /(1-s)}{\widetilde{r}} .
$$

12 As pointed out in [42], the proof in [9] needs a small correction, which yields the fractional chain rule in a less general context. See $[25,42,44]$. 
By applying (3.9), the product estimate (3.5) in Lemma 3.1 (with sufficiently small $\alpha>0$ ), and Lemma 3.2, there exists $\theta>0$ such that

$$
\begin{aligned}
\left\|\operatorname{Im}\left(\Theta_{N} e^{i \beta v_{N}}\right)\right\|_{N^{s}(T)} & \lesssim\left\|\operatorname{Im}\left(\Theta_{N} e^{i \beta v_{N}}\right)\right\|_{L_{T}^{\widetilde{q}_{1}} W_{x}^{-\alpha, \widetilde{r}_{1}}} \\
& \lesssim T^{\theta}\left\|\Theta_{N}\right\|_{L_{T}^{q_{2}} W_{x}^{-\alpha, \infty}\left\|e^{i \beta v_{N}}\right\|_{L_{T}^{\widetilde{q}_{2}} W_{x}^{\alpha, \widetilde{r}_{2}}}} \\
& \lesssim T^{\theta}\left\|\Theta_{N}\right\|_{L_{T}^{q_{2}} W_{x}^{-\alpha, \infty}}\left(1+\beta\left\|v_{N}\right\|_{L_{T}^{\widetilde{q}_{2}} W_{x}^{\alpha, \widetilde{r}_{2}}}\right)
\end{aligned}
$$

for any finite $\widetilde{q}_{2}>\widetilde{q}_{1}$ and $\widetilde{r}_{2}>\widetilde{r}_{1}$ with $\frac{1}{\widetilde{q}_{1}}>\frac{1}{q_{2}}+\frac{1}{\widetilde{q}_{2}}$.

It follows from (3.10) and (3.11) that by taking $\alpha \rightarrow 0$, we can take $\left(q_{1}, r_{1}\right)$ (and $\left(\widetilde{q}_{1}, \widetilde{r}_{1}\right)$, respectively) arbitrarily close to $(q, r)$ (and to $(\widetilde{q}, \widetilde{r})$, respectively). Moreover, by taking $\alpha>0$ sufficiently small, we can also take $\left(\widetilde{q}_{2}, \widetilde{r}_{2}\right)$ arbitrarily close to $\left(\widetilde{q}_{1}, \widetilde{r}_{1}\right)$. Hence, from (3.1), we can guarantee

$$
2 \widetilde{q}_{2}<q_{1} \text { and } 2 \widetilde{r}_{2}<r_{1}
$$

by taking $\alpha>0$ sufficiently small. Therefore, from (3.8) and (3.12) with (3.9) and (3.13), we obtain

$$
\left\|\Phi^{N}\left(v_{N}\right)\right\|_{X^{s}(T)} \lesssim\left\|\left(u_{0}, u_{1}\right)\right\|_{\mathcal{H}^{s}}+T^{\theta}\left\|\Theta_{N}\right\|_{L_{T}^{q_{2}} W_{x}^{-\alpha, \infty}}\left(1+\beta\left\|v_{N}\right\|_{X^{s}(T)}\right) .
$$

Proceeding as in (3.8) and (3.12), we have

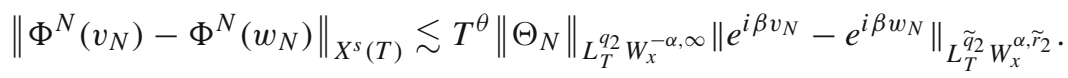

As for the last factor in (3.15), by applying the mean value theorem with $F(u)=$ $e^{i \beta u}$, the fractional Leibniz rule (3.4) in Lemma 3.1, and the fractional chain rule (Lemma 3.2), we have

$$
\begin{aligned}
& \left\|e^{i \beta v_{N}}-e^{i \beta w_{N}}\right\|_{L_{T}^{\widetilde{q}_{2}} W_{x}^{\alpha, \widetilde{r}_{2}}} \\
& =\left\|\left(v_{N}-w_{N}\right) \int_{0}^{1} F^{\prime}\left(\tau v_{N}+(1-\tau) w_{N}\right) d \tau\right\|_{L_{T}^{\widetilde{q}_{2}} W_{x}^{\alpha, \tilde{r}_{2}}}
\end{aligned}
$$

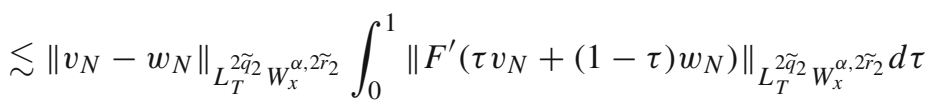

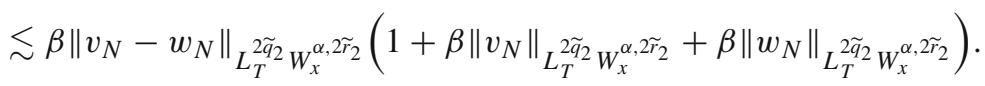

Hence, from (3.15) and (3.16), we obtain

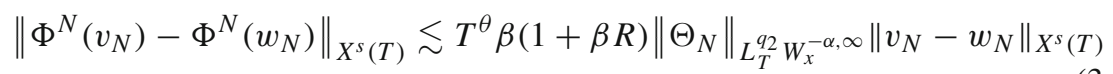


for $v_{N}, w_{N} \in B_{R} \subset X^{s}(T)$.

Let $\alpha>0$ be sufficiently small as above. Then, Proposition 1.1 states that, given any finite $p \geq 1,\left\{\Theta_{N}\right\}_{N \in \mathbb{N}}$ is uniformly bounded in $L^{p}\left(\Omega ; L^{q_{2}}\left(\left[0, T_{0}\right] ; W^{-\alpha, \infty}\left(\mathbb{T}^{2}\right)\right)\right)$, provided that

$$
0<T_{0}<\frac{8 \pi \alpha}{\beta^{2}}
$$

Fix $T_{0}>0$ satisfying (3.18). Given $N \in \mathbb{N}$ and $\lambda>0$, define $\Omega_{N, \lambda}$ by

$$
\Omega_{N, \lambda}=\left\{\omega \in \Omega:\left\|\Theta_{N}\right\|_{L_{T_{0}}^{q_{2}} W_{x}^{-\alpha, \infty}}<\lambda\right\}
$$

On $\Omega_{N, \lambda}$, it follows from (3.14) and (3.17) that $\Phi^{N}$ is a contraction on the ball $B_{R} \subset$ $X^{s}(T)$, where $R \sim\left\|\left(u_{0}, u_{1}\right)\right\|_{\mathcal{H}^{s}}$ and $T=T(R, \lambda, \beta)=T\left(\left\|\left(u_{0}, u_{1}\right)\right\|_{\mathcal{H}^{s}}, \lambda, \beta\right)>0$ such that $T \leq T_{0}$. Note that we can choose $T>0$ such that

$$
T \sim_{R, \beta}(1+\lambda)^{-\kappa}
$$

for some $\kappa>0$ and any $\lambda \gg 1$.

By Proposition 1.1 and Chebyshev's inequality, we have

$$
\sup _{N \in \mathbb{N}} P\left(\Omega_{N, \lambda}^{c}\right)=o(1)
$$

as $\lambda \rightarrow \infty$. This proves local well-posedness of (3.7) uniformly in $N \in \mathbb{N}$. Here, the uniformity refers to the fact that, given any small $T>0$, we have a uniform (in $N$ ) control (i.e. a uniform lower bound in terms of $T$ ) on the probabilities of the sets $\Omega_{N}(T)=\Omega_{N, \lambda(T)}$, where well-posedness of (3.7) holds on [0,T], thanks to (3.19) and (3.20).

Let $\Theta$ be the limit of $\Theta_{N}$ in $L^{p}\left(\Omega ; L^{q_{2}}\left(\left[0, T_{0}\right] ; W^{-\alpha, \infty}\left(\mathbb{T}^{2}\right)\right)\right)$ constructed in Proposition 1.1. Define $\Omega_{\lambda}$ by

$$
\Omega_{\lambda}=\left\{\omega \in \Omega:\|\Theta\|_{\left.L_{T_{0}}^{q_{2}} W_{x}^{-\alpha, \infty}<\lambda\right\}}\right.
$$

Then, by repeating the argument above, we see that the limiting equation (1.20) for $v$, written in the Duhamel formulation as

$$
v(t)=\partial_{t} S(t) u_{0}+S(t) u_{1}-\int_{0}^{t} S\left(t-t^{\prime}\right) \operatorname{Im}\left(\Theta e^{i \beta v}\right)\left(t^{\prime}\right) d t^{\prime},
$$

is well-posed on the time interval [0,T], where $T=T(R, \lambda, \beta)<T_{0}$ satisfies (3.19). In view of Proposition 1.1, we then conclude that there exists an almost surely positive stopping time

$$
\tau=\tau\left(\left\|\left(u_{0}, u_{1}\right)\right\|_{\mathcal{H}^{s}}, \beta\right) \sim C\left(\left\|\left(u_{0}, u_{1}\right)\right\|_{\mathcal{H}^{s}}, \beta\right)\left(1+\|\Theta\|_{L_{T_{0}}^{q_{2}} W_{x}^{-\alpha, \infty}}\right)^{-\kappa}
$$


such that the limiting equation (1.20) for $v$ is well-posed on the time interval $[0, \tau]$.

In the following, fix sufficiently small $T=T\left(\left\|\left(u_{0}, u_{1}\right)\right\|_{\mathcal{H}^{s}}, \beta\right)>0$, satisfying (3.18) (with $T_{0}$ replaced by $T$ ) and set $\Omega(T)=\{\tau \geq T\}$, where $\tau$ is defined in (3.23). Namely, for any $\omega \in \Omega(T)$, the limiting equation (3.22) is well-posed on $[0, T]$ by the argument above. Moreover, in view of (3.21) and (3.23), we may assume that $\Omega(T)=\Omega_{\lambda}$ for some $\lambda=\lambda(T)>0$ satisfying (3.19). Note that $P(\Omega(T))>0$ for any sufficiently small $T>0$ and $P(\Omega(T)) \rightarrow 1$ as $T \rightarrow 0$. In the following, we work with the conditional probability $P_{T}$ given the event $\Omega(T)$, defined by

$$
P_{T}(A)=P(A \mid \Omega(T))=\frac{P(A \cap \Omega(T))}{P(\Omega(T))} .
$$

We first check that the truncated dynamics (3.7) is well-posed on $[0, T]$ outside a set of $P_{T}$-probability $o(1)$ as $N \rightarrow \infty$. Let $N \in \mathbb{N}$. By defining a set $\Sigma_{N}$ by

$$
\Sigma_{N}=\left\{\omega \in \Omega:\left\|\Theta_{N}-\Theta\right\|_{L_{T_{0}}^{q_{2}} W_{x}^{-\alpha, \infty}} \leq 1\right\}
$$

we have

$$
\Omega(T) \cap \Sigma_{N}=\Omega_{\lambda} \cap \Sigma_{N} \subset \Omega_{N, \lambda+1}
$$

Without loss of generality, we may assume that the Eq. (3.7) is also well-posed on $[0, T]$ for any $\omega \in \Omega(T) \cap \Sigma_{N}$. Furthermore, since $\Theta_{N}$ converges in probability to $\Theta$ in $L^{q_{2}}\left([0, T] ; W^{-\alpha, \infty}\left(\mathbb{T}^{2}\right)\right)$, we have $P\left(\Sigma_{N}^{c}\right) \rightarrow 0$ as $N \rightarrow \infty$. Namely, we have

$$
P_{T}\left(\Omega(T) \cap \Sigma_{N}\right) \longrightarrow 1
$$

as $N \rightarrow \infty$. This verifies well-posedness of the truncated dynamics (3.7) on the time interval $[0, T]$ asymptotically $P_{T}$-almost surely in the sense of (3.24).

Given $\left(u_{0}, u_{1}\right) \in \mathcal{H}^{s}\left(\mathbb{T}^{2}\right)$ and $\left.\Theta_{0} \in L^{q_{2}}\left([0, T] ; W^{-\alpha, \infty}\left(\mathbb{T}^{2}\right)\right)\right)$, define a map $\Phi=$ $\Phi_{\left(u_{0}, u_{1}\right), \Theta_{0}}$ by

$$
\Phi(v)(t)=\partial_{t} S(t) u_{0}+S(t) u_{1}-\int_{0}^{t} S\left(t-t^{\prime}\right) \operatorname{Im}\left(\Theta_{0} e^{i \beta v}\right)\left(t^{\prime}\right) d t^{\prime} .
$$

Then, a slight modification of the analysis presented above shows that the map:

$$
\left.\left(\left(u_{0}, u_{1}\right), \Theta_{0}\right) \in \mathcal{H}^{s}\left(\mathbb{T}^{2}\right) \times L^{q_{2}}\left([0, T] ; W^{-\alpha, \infty}\left(\mathbb{T}^{2}\right)\right)\right) \longrightarrow v \in X^{s}(T)
$$

is continuous, where $v=\Phi(v)$ is the unique fixed point for $\Phi$ and $0<T \leq T_{0}$ is sufficiently small. From this observation and convergence in probability of $\Theta_{N}$ to $\Theta$ deduced from Proposition 1.1, we conclude that the solution $v_{N}$ to (3.25) converges in probability with respect to the conditional probability $P_{T}$ to $v$ in $X^{s}(T) .{ }^{13}$

\footnotetext{
13 Note that the truncated equation (3.7) may not be well-posed on the time interval [0,T], $P_{T}$-almost surely. This, however, does not cause a problem since, as verified in (3.24), the truncated equation (3.7) is well-posed on $[0, T]$ asymptotically $P_{T}$-almost surely.
} 
Finally, recalling the decompositions

$$
u_{N}=\Psi_{N}+v_{N} \text { and } u=\Psi+v
$$

and from Lemma 2.6 that $\Psi_{N}$ converges $P$-almost surely (and hence $P_{T}$-almost surely) to $\Psi$ in $C\left([0, T] ; W^{-\varepsilon, \infty}\left(\mathbb{T}^{2}\right)\right)$, we conclude that $u_{N}$ converges to $u$, in $C\left([0, T] ; W^{-\varepsilon, \infty}\left(\mathbb{T}^{2}\right)\right)$, in probability with respect to the conditional probability $P_{T}$. This completes the proof of Theorem 1.2.

\subsection{Triviality of the unrenormalized model}

We conclude this paper by establishing a triviality result for the unrenormalized model (Proposition 1.4), whose proof follows from a small modification of the proof of Theorem 1.2.

The main idea is to follow the idea from $[23,33,35]$ and artificially introduce a renormalization constant $\gamma_{N}(t)$ in (1.18). Note that unlike the previous work [23,33, 35 ], our renormalization constant appears in a multiplicative manner, which makes our analysis particularly simple. Start with the truncated equation (1.21) and rewrite it as

$$
\partial_{t}^{2} u_{N}+(1-\Delta) u_{N}+\gamma_{N}^{-1} \operatorname{Im}\left(\gamma_{N} e^{i \beta u_{N}}\right)=\mathbf{P}_{N} \xi
$$

With the decomposition

$$
u_{N}=\Psi_{N}+v_{N}
$$

it follows from (1.16) and (1.18) that $\gamma_{N} e^{i \beta u_{N}}=\Theta_{N} e^{i \beta v_{N}}$. Then, we may repeat the analysis presented in the previous subsection and establish local well-posedness for the equation satisfied by $v_{N}$ :

$$
\left\{\begin{array}{l}
\partial_{t}^{2} v_{N}+(1-\Delta) v_{N}+\gamma_{N}^{-1} \operatorname{Im}\left(\Theta_{N} e^{i \beta v_{N}}\right)=0 \\
\left.\left(v_{N}, \partial_{t} v_{N}\right)\right|_{t=0}=\left(u_{0}, u_{1}\right)
\end{array}\right.
$$

Noting that $0 \leq \gamma_{N}^{-1}(t) \leq 1$, we once again have a uniform (in $N$ ) control on the probabilities of the sets $\Omega_{N}(T)=\Omega_{N, \lambda(T)}$, where well-posedness of (3.27) holds on $[0, T]$. Moreover, we have

$$
\left\|v_{N}\right\|_{X^{s}(T)} \lesssim\left\|\left(u_{0}, u_{1}\right)\right\|_{\mathcal{H}^{s}}
$$

on $\Omega_{N}(T)$.

By writing (3.27) in the Duhamel formulation:

$$
v_{N}(t)=\partial_{t} S(t) u_{0}+S(t) u_{1}-\int_{0}^{t} S\left(t-t^{\prime}\right) \gamma_{N}^{-1}\left(t^{\prime}\right) \operatorname{Im}\left(\Theta_{N} e^{i \beta v_{N}}\right)\left(t^{\prime}\right) d t^{\prime},
$$


we see from (3.8) and (3.12) with (3.28) that

$$
\begin{aligned}
\left\|v_{N}-\partial_{t} S(t) u_{0}-S(t) u_{1}\right\|_{X^{s}(T)} \lesssim & T^{\theta}\left\|\gamma_{N}^{-1}(t) \Theta_{N}\right\|_{L_{T}^{q_{2}} W_{x}^{-\alpha, \infty}}\left(1+\beta\left\|v_{N}\right\|_{X^{s}(T)}\right) \\
\lesssim & \left\|\gamma_{N}^{-1}(t)\right\|{ }_{\frac{1}{\delta}}\left\|\Theta_{N}\right\|_{L_{T_{0}}^{\frac{q_{2}}{1-\delta q_{2}}} W_{x}^{-\alpha, \infty}} \\
& \times\left(1+\beta\left\|\left(u_{0}, u_{1}\right)\right\|_{\mathcal{H}^{s}}\right)
\end{aligned}
$$

for any small $\delta>0$. From (1.18) with (1.12) and Lemma 2.7, we have

$$
\left\|\gamma_{N}^{-1}(t)\right\|_{L_{T}^{\frac{1}{\delta}}} \lesssim\left\|e^{-\frac{\beta^{2} t}{8 \pi} \log N}\right\|_{L_{T}^{\frac{1}{\delta}}} \leq C(\beta, \delta)(\log N)^{-1} \longrightarrow 0
$$

as $N \rightarrow \infty$. Then, it follows from (3.29), (3.30), Proposition 1.1, and Chebyshev's inequality that $v_{N}-\partial_{t} S(t) u_{0}-S(t) u_{1}$ converges in probability to 0 in $X^{s}(T)$ as $N \rightarrow \infty$. Recalling the decomposition (3.26) and the almost sure convergence of $\Psi_{N}$ to $\Psi$ in $C\left([0, T] ; W^{-\varepsilon, \infty}\left(\mathbb{T}^{2}\right)\right.$ ) (Lemma 2.6), we conclude that $u_{N}$ converges in probability to $\partial_{t} S(t) u_{0}+S(t) u_{1}+\Psi$, which is the unique solution to the linear stochastic wave equation (1.22) with initial data $\left(u_{0}, u_{1}\right)$. This completes the proof of Proposition 1.4.

Acknowledgements T.O. and T.R. were supported by the European Research Council (Grant No. 637995 "ProbDynDispEq"). P.S. was partially supported by NSF Grant DMS-1811093. The authors would like to thank the anonymous referees for the helpful comments.

Open Access This article is licensed under a Creative Commons Attribution 4.0 International License, which permits use, sharing, adaptation, distribution and reproduction in any medium or format, as long as you give appropriate credit to the original author(s) and the source, provide a link to the Creative Commons licence, and indicate if changes were made. The images or other third party material in this article are included in the article's Creative Commons licence, unless indicated otherwise in a credit line to the material. If material is not included in the article's Creative Commons licence and your intended use is not permitted by statutory regulation or exceeds the permitted use, you will need to obtain permission directly from the copyright holder. To view a copy of this licence, visit http://creativecommons.org/licenses/by/4.0/.

\section{References}

1. Albeverio, S., Haba, Z., Russo, F.: Trivial solutions for a nonlinear two-space-dimensional wave equation perturbed by space-time white noise. Stochastics 56(1-2), 127-160 (1996)

2. Albeverio, S., Haba, Z., Russo, F.: A two-space dimensional semilinear heat equation perturbed by (Gaussian) white noise. Probab. Theory Relat. Fields 121(3), 319-366 (2001)

3. Aronszajn, N., Smith, K.: Theory of Bessel potentials. I. Ann. Inst. Fourier (Grenoble) 11, 385-475 (1961)

4. Barone, A., Esposito, F., Magee, C., Scott, A.: Theory and applications of the sine-Gordon equation. Rivista del Nuovo Cimento 1, 227-267 (1971)

5. Bényi, Á., Oh, T.: The Sobolev inequality on the torus revisited. Publ. Math. Debrecen 83(3), 359-374 (2013)

6. Bourgain, J.: Periodic nonlinear Schrödinger equation and invariant measures. Commun. Math. Phys. 166, 1-26 (1994) 
7. Bourgain, J.: Invariant measures for the 2D-defocusing nonlinear Schrödinger equation. Commun. Math. Phys. 176, 421-445 (1996)

8. Chandra, A., Hairer, M., Shen, H.: The dynamical sine-Gordon model in the full subcritical regime. arXiv: 1808.02594 [math.PR]

9. Christ, M., Weinstein, M.: Dispersion of small amplitude solutions of the generalized Korteweg-de Vries equation. J. Funct. Anal. 100, 87-109 (1991)

10. Da Prato, G., Debussche, A.: Strong solutions to the stochastic quantization equations. Ann. Probab. 31(4), 1900-1916 (2003)

11. Da Prato, G., Zabczyk, J.: Stochastic equations in infinite dimensions. In: Encyclopedia of Mathematics and its Applications, vol. 152 (2nd edn), pp. xviii+493. Cambridge University Press, Cambridge (2014)

12. E. W., Jentzen, A., Shen, H.: Renormalized powers of Ornstein-Uhlenbeck processes and wellposedness of stochastic Ginzburg-Landau equations. Nonlinear Anal. 142, 152-193 (2016)

13. Fröhlich, J.: Classical and quantum statistical mechanics in one and two dimensions: two-component Yukawa- and Coulomb systems. Commun. Math. Phys. 47(3), 233-268 (1976)

14. Garban, C.: Dynamical Liouville. J. Funct. Anal. 278(6), 108351 (2020)

15. Gatto, A.E.: Product rule and chain rule estimates for fractional derivatives on spaces that satisfy the doubling condition. J. Funct. Anal. 188(1), 27-37 (2002)

16. Ginibre, J., Velo, G.: Generalized Strichartz inequalities for the wave equation. J. Funct. Anal. 133, 50-68 (1995)

17. Grafakos, L.: Classical Fourier analysis. In: Graduate Texts in Mathematics, vol. 249 (3rd edn), pp. xviii+638. Springer, New York (2014)

18. Grafakos, L.: Modern Fourier analysis. In: Graduate Texts in Mathematics, vol. 250 (3rd edn), pp. xvi+624. Springer, New York (2014)

19. Gubinelli, M., Koch, H., Oh, T.: Renormalization of the two-dimensional stochastic nonlinear wave equation. Trans. Am. Math. Soc. 370, 7335-7359 (2018)

20. Gubinelli, M., Koch, H., Oh, T.: Paracontrolled approach to the three-dimensional stochastic nonlinear wave equation with quadratic nonlinearity. arXiv:1811.07808 [math.AP]

21. Gubinelli, M., Koch, H., Oh, T., Tolomeo, L.: Global dynamics for the two-dimensional stochastic nonlinear wave equations. Preprint

22. Hairer, M.: A theory of regularity structures. Invent. Math. 198, 269-504 (2014)

23. Hairer, M., Ryser, M.D., Weber, H.: Triviality of the 2D stochastic Allen-Cahn equation. Electron. J. Probab. 17(39), 14 (2012)

24. Hairer, M., Shen, H.: The dynamical sine-Gordon model. Commun. Math. Phys. 341(3), 933-989 (2016)

25. Kato, T.: On nonlinear Schrödinger equations. II. $H^{s}$-solutions and unconditional well-posedness. J. Anal. Math. 67, 281-306 (1995)

26. Keel, M., Tao, T.: Endpoint Strichartz estimates. Am. J. Math. 120(5), 955-980 (1998)

27. Killip, R., Stovall, B., Visan, M.: Blowup behaviour for the nonlinear Klein-Gordon equation. Math. Ann. 358(1-2), 289-350 (2014)

28. Lacoin, H., Rhodes, R., Vargas, V.: Complex Gaussian multiplicative chaos. Commun. Math. Phys. 337(2), 569-632 (2015)

29. Lacoin, H., Rhodes, R., Vargas, V.: A probabilistic approach of ultraviolet renormalisation in the boundary sine-Gordon model. arXiv:1903.01394 [math.PR]

30. Lindblad, H., Sogge, C.: On existence and scattering with minimal regularity for semilinear wave equations. J. Funct. Anal. 130, 357-426 (1995)

31. McKean, H.P.: The sine-Gordon and sinh-Gordon equations on the circle. Commun. Pure Appl. Math. 34(2), 197-257 (1981)

32. McKean, H.P., Vaninsky, K.L.: Statistical mechanics of nonlinear wave equations. In: Trends and Perspectives in Applied Mathematics (Applied Mathematical Sciences, vol. 100), pp. 239-264. Springer, New York (1994)

33. Oh, T., Okamoto, M., Robert, T.: A remark on triviality for the two-dimensional stochastic nonlinear wave equation. arXiv:1905.06278 [math.AP]

34. Oh, T., Okamoto, M., Tzvetkov, N.: Uniqueness and non-uniqueness of the Gaussian free field evolution under the two-dimensional Wick ordered cubic wave equation. Preprint

35. Oh, T., Pocovnicu, O., Tzvetkov, N.: Probabilistic local well-posedness of the cubic nonlinear wave equation in negative Sobolev spaces. arXiv:1904.06792 [math.AP] 
36. Oh, T., Robert, T., Sosoe, P., Wang, Y.: Invariant Gibbs dynamics for the dynamical sine-Gordon model. arXiv:2001.09275 [math.AP]

37. Oh, T., Robert, T., Tzvetkov, N.: Stochastic nonlinear wave dynamics on compact surfaces. arXiv:1904.05277 [math.AP]

38. Oh, T., Robert, T., Wang, Y.: On the parabolic and hyperbolic Liouville equations. arXiv:1908.03944 [math.AP]

39. Oh, T., Thomann, L.: Invariant Gibbs measures for the 2- $d$ defocusing nonlinear wave equations. Ann. Fac. Sci. Toulouse Math. (to appear)

40. Perring, J., Skyrme, T.: A model unified field equation. Nucl. Phys. 31, 550-555 (1962)

41. Ryang, S., Saito, T., Shigemoto, K.: Canonical stochastic quantization. Progr. Theoret. Phys. 73(5), 1295-1298 (1985)

42. Staffilani, G.: The initial value problem for some dispersive differential equations. Thesis (Ph.D.) The University of Chicago (1995)

43. Sun, C., Tzvetkov, N.: New examples of probabilistic well-posedness for nonlinear wave equations. J. Funct. Anal. 278(2), 108322 (2020)

44. Taylor, M.: Tools for PDE, pseudodifferential operators, paradifferential operators, and layer potentials. In: Mathematical Surveys and Monographs, vol. 81. American Mathematical Society, Providence, RI (2000)

Publisher's Note Springer Nature remains neutral with regard to jurisdictional claims in published maps and institutional affiliations. 